\title{
Aeroacoustics of the swinging corrugated tube: Voice of the Dragon
}

\author{
Güneş Nakiboğlu, ${ }^{\text {a) }}$ Oleksii Rudenko, and Avraham Hirschberg \\ Eindhoven University of Technology, Department of Applied Physics, Laboratory of Fluid Mechanics, \\ P.O. Box 513, 5600 MB Eindhoven, The Netherlands
}

(Received 11 December 2010; revised 13 April 2011; accepted 14 April 2011)

\begin{abstract}
When one swings a short corrugated pipe segment around one's head, it produces a musically interesting whistling sound. As a musical toy it is called a "Hummer" and as a musical instrument, the "Voice of the Dragon." The fluid dynamics aspects of the instrument are addressed, corresponding to the sound generation mechanism. Velocity profile measurements reveal that the turbulent velocity profile developed in a corrugated pipe differs notably from the one of a smooth pipe. This velocity profile appears to have a crucial effect both on the non-dimensional whistling frequency (Strouhal number) and on the amplitude of the pressure fluctuations. Using a numerical model based on incompressible flow simulations and vortex sound theory, excellent predictions of the whistling Strouhal numbers are achieved. The model does not provide an accurate prediction of the amplitude. In the second part of the paper the sound radiation from a Hummer is discussed. The acoustic measurements obtained in a semi-anechoic chamber are compared with a theoretical radiation model. Globally the instrument behaves as a rotating (Leslie) horn. The effects of Doppler shift, wall reflections, bending of the tube, non-constant rotational speed on the observed frequency, and amplitude are discussed. (C) 2012 Acoustical Society of America. [DOI: 10.1121/1.3651245]
\end{abstract}

PACS number(s): 43.28.Py, 43.75.Np, 43.75.Qr, 43.75.Ef [TRM] Pages: 749-765

\section{INTRODUCTION}

In thin walled pipes corrugations provide local stiffness while allowing for a global flexibility. This makes corrugated pipes convenient for various industrial applications ranging from vacuum cleaners to offshore natural gas production. ${ }^{1}$ Flow through this type of pipes can sustain high amplitude whistling tones, which do not occur in smooth pipes. This whistling is an environmental annoyance and associated vibration can lead to mechanical failure. ${ }^{2}$

Short corrugated pipe segments are also used as musical toys and instruments. The "Hummer" 3 is a flexible plastic corrugated pipe of approximately $75 \mathrm{~cm}$ length and $3 \mathrm{~cm}$ diameter, as shown in Fig. 1. While holding one end by swinging the tube around the head, various tones can be produced. This chorus like sound is musically interesting. The instrument has received the names "Voice of the Dragon",4 and "Lasso d'Amore."

A more extensive review of the literature on corrugated pipes is given in the earlier papers of the authors. ${ }^{7,8}$ Physical modeling of corrugated pipes by means of simple source models placed along a tube has been proposed by Debut ${ }^{9}$ and Goyder. ${ }^{10}$ A Large eddy simulation has been attempted by Popescu and Johansen, ${ }^{11}$ but results seem to be in contradiction with the experimental studies. ${ }^{8,12,13}$

In the present paper the physical modeling of this instrument is discussed. In the next section, an overview of the basic principles is given. The following two sections focus on the flow and the associated sound production within the

\footnotetext{
a) Author to whom correspondence should be addressed. Electronic mail: g.nakiboglu@tue.nl
}

tube. The fourth section considers the radiation of the sound from the open pipe terminations. The fifth section covers some of the mystery that is removed and remaining open questions. The last section concludes the study.

\section{BASIC PRINCIPLES}

The whistling of the Hummer is induced by the flow through the pipe driven by its rotation. This can be demonstrated by closing the stationary pipe termination, which is held with the hand. Placing the thumb in the tube or covering the entrance with the palm are convenient ways to do so. This suppresses the whistling. Another way to demonstrate that it is the flow through the corrugated pipe that sustains the whistling, is to blow through the pipe. Our lung capacity is not sufficient to make a typical Hummer whistle. However, one can take a narrower corrugated pipe and make it whistle. A corrugated pipe with a diameter of $D=1 \mathrm{~cm}$ and a length of $L=1 \mathrm{~m}$ used as a protection jacket for electrical cables in buildings, whistles nicely at a rather high pitch.

In flows producing sound the fluid velocities are so high that the pressure forces are mainly balanced by the inertia of the fluid. The viscous forces are negligible in the bulk of the flow. They only become important within thin boundary layers close to the wall. The pressure in these boundary layers is imposed by the main flow. ${ }^{14}$ In the boundary layer due to viscous losses a fluid particle does not have enough kinetic energy to travel against an adverse pressure gradient, as it would do in the bulk of the flow. This results in a back flow along the wall opposite to the main flow direction and ultimately a separation of the boundary layer from the wall at an abrupt pipe widening. This forms a so-called shear layer, separating the high speed bulk flow region from the 


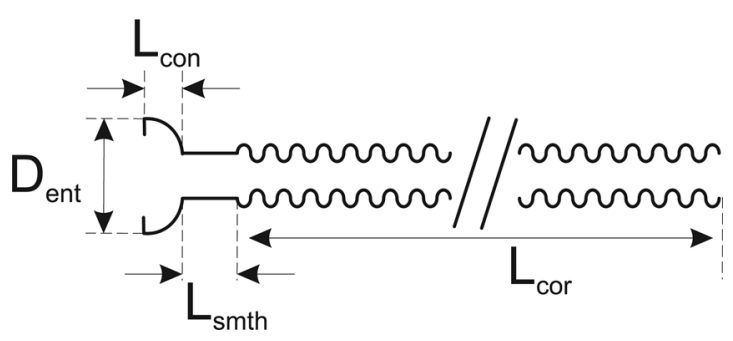

FIG. 1. A schematic drawing of a Hummer.

low speed flow region close to the walls. This separation occurs at each corrugation, leaving almost a stagnant fluid in the cavities. These shear layers are quite unstable and the resulting unsteadiness of the flow is a source of sound. ${ }^{15}$ Furthermore the flow separation is also very sensitive to acoustical perturbations. These perturbations trigger the rollup of the shear layer into vortices. This receptivity of the shear layer to acoustic perturbations is essential in the whistling process. It couples the vortex shedding developing at each corrugation with the global standing acoustical wave in the tube. As a consequence, the unsteadiness of the flow within each cavity (corrugation) along the pipe is synchronized with a global acoustic oscillation of the pipe. Actually, it is a feedback system in which the flow instability at each cavity is a power supply and the pipe is a filter, selecting a specific tone corresponding to a standing longitudinal wave (resonance mode). This is a sound amplification by simulated emission radiation device analogous to a laser. Such a feedback system can produce a periodic oscillation only if there is a non-linear saturation mechanism, which limits the amplitude. $^{16}$

\section{FLUID DYNAMICS}

\section{A. Frictionless model}

The average flow velocity $U_{\text {avrg }}$ through the swinging pipe can be estimated by assuming a steady frictionless flow. As the velocities are low compared to the speed of sound, the pressure difference across the pipe is very small compared to the atmospheric pressure. One can therefore neglect the density variation in the steady component of the flow. The fact that the air is almost incompressible implies that, in a steady flow, the volume flux $Q$ along the tube must be independent of the position $x$ along the tube, measured from the fixed open end. If we neglect changes in the shape of the velocity profile $U(r)$, with $r$ is the distance from the pipe axis, the flow velocity remains constant along the pipe. This velocity is defined by $U_{\mathrm{avrg}}=4 Q /\left(\pi D^{2}\right)$. Because of the swinging motion, the tube is rotating with an angular velocity $\Omega$. A fluid particle, corresponding to a slice of the tube of length $d x$, will undergo a centrifugal force $\rho_{0}\left(\pi d x D^{2} / 4\right) \Omega^{2} x$, where $\rho_{0}=1.2 \mathrm{~kg} / \mathrm{m}^{3}$ is the air density. As the fluid velocity is constant, this force should be balanced by the pressure forces $-[p(x+d x)-p(x)] \pi D^{2} / 4=-d p\left(\pi D^{2} / 4\right)$. This yields the differential equation for the pressure $p$ :

$$
d p=\rho_{0} \Omega^{2} x d x
$$

Integration between the stationary tube inlet $x=0$ and the moving tube outlet $x=L$ yields

$$
p(L)-p(0)=\frac{1}{2} \rho_{0} \Omega^{2} L^{2}
$$

Note that this equation has the opposite sign from the equation used by Silverman and Cushman ${ }^{4}$ and Serafin and Kojs. ${ }^{5}$ This is due to the fact that Silverman and Cushman ${ }^{4}$ ignored the impact of the centrifugal force on their measurement of the pressure difference and made the erroneous assumption that the inlet pressure $p(0)$ should be equal to atmospheric pressure $p_{\text {atm }}$. In fact, as a result of flow separation, a free jet is formed at the swinging outlet of the pipe. Like in the plume flowing out of a chimney, the pressure $p(L)$ in this free jet is equal to the surrounding atmospheric pressure $p_{\text {atm. }}{ }^{17}$ The low pressure at the inlet,

$$
p(0)=p_{\mathrm{atm}}-\frac{1}{2} \rho_{0} \Omega^{2} L^{2},
$$

is actually sucking the surrounding air into the pipe. This explains the observation of Silverman and Cushman ${ }^{4}$ that small bits of tissue paper placed in the palm will be sucked up into the tube and discharged from the rotating end. Assuming a steady incompressible frictionless flow around the inlet, one finds from the conservation of mechanical energy (Bernoulli):

$$
p_{\mathrm{atm}}=p(0)+\frac{1}{2} \rho_{0} U_{\mathrm{avrg}}^{2},
$$

which combined with Eq. (3) yields the very simple result:

$$
U_{\mathrm{avrg}}=\Omega L
$$

In this simple model the friction is neglected (except for flow separation at the outlet), which leads to a uniform velocity profile in the pipe. In reality, however, as a result of friction the velocity in the pipe will be lower near the walls than in the middle, so that a non-uniform velocity profile will develop. The shape of the velocity profile is expected to be important in corrugated pipes both for the frequency and the amplitude of the whistling. ${ }^{7}$ In Sec. III C 1 , the velocity profile in a Hummer is addressed.

\section{B. Experimental setup}

The velocity profile in a Hummer was determined by means of hotwire measurements. Figure 2 shows the experimental setup. An aluminum pipe with a diameter of $33 \mathrm{~mm}$ and a length of $60 \mathrm{~mm}$ was inserted to the conical section at the inlet of the Hummer (Fig. 1). Using a clamp for standard vacuum appliances (ISO-KF), the aluminum pipe was attached to the settling chamber of the wind tunnel in an airtight manner. The settling chamber is a wooden box of $0.5 \mathrm{~m} \times 0.5 \mathrm{~m} \times 1.8 \mathrm{~m}$. The flow is driven by a centrifugal ventilator. An $8 \mathrm{~cm}$ thick layer of acoustic foam on the inner walls of the settling chamber prevents acoustical resonances of the box. The Hummer lay on a horizontal table and passed 


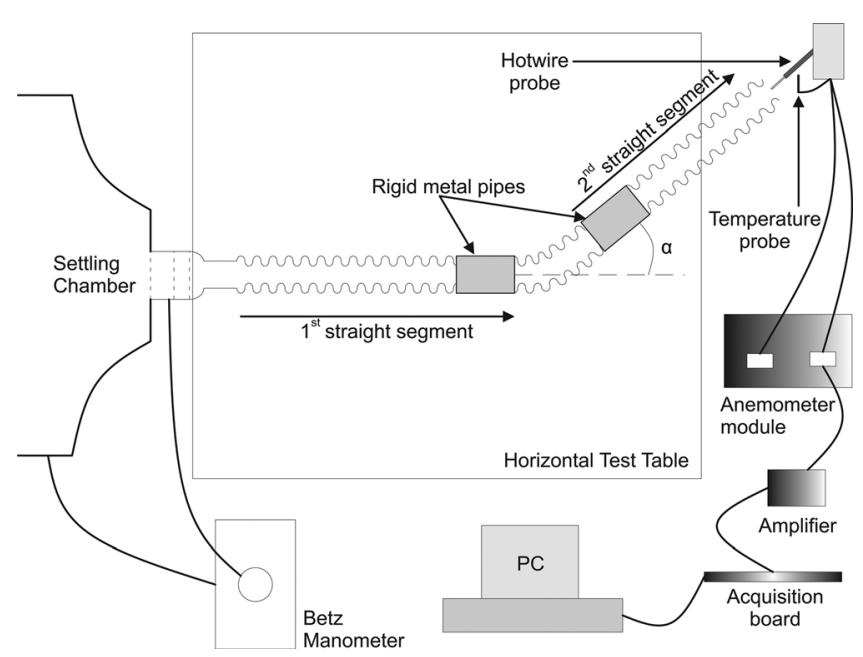

FIG. 2. Experimental setup.

through two rigid metal pipe segments with a diameter of $33 \mathrm{~mm}$ and length of $100 \mathrm{~mm}$. Using these rigid pipe segments the Hummer was fixed on the table without pressing on the elastic plastic walls. Also by changing the position of the second rigid pipe, the Hummer could be bent in the horizontal plane. The effect of the bending is addressed in Sec. VI B, all the other results that are presented were obtained with a straight Hummer.

The average velocity $\left(U_{\text {avrg }}\right)$ was calculated from the pressure difference across the inlet contraction using the equation of Bernoulli [Eq. (4)]. The pressure difference is measured by means of a Betz micromanometer. The velocity profile at the end of the Hummer was measured with a hotwire probe (Dantec probe type 55P11). The hotwire anemometer used in this study was a Dantec 90C10 CTA module installed within a Dantec 90N10 frame. The signal was amplified and low-pass filtered through a low-noise preamplifier (Stanford Research Systems, Model SR560) and sent to the computer via a National Instrument BNC-2090 data acquisition board with a 12-bit resolution at a sampling rate of $10 \mathrm{kHz}$. A sampling duration of 10 second was used for each position. All the data were obtained using the Dantec StreamWare software. The hotwire signals were compensated for variations in the flow temperature. Integration of the velocity profile over the outlet provides a second measurement for the flow velocity $U_{\text {avrg }}$.

Experiments were performed on a Hummer manufactured by Jono Toys, b.v., Holland. The Hummer has a corrugated length of $L_{\text {cor }}=700 \mathrm{~mm}$, a smooth length of $L_{\text {smth }}=30 \mathrm{~mm}$, a conical section length of $L_{\mathrm{con}}=10 \mathrm{~mm}$ and an entrance diameter $D_{\text {ent }}=33 \mathrm{~mm}$, as shown in Fig. 1. The remaining geometric parameters are shown in Fig. 3, where only a few corrugations are sketched. The wave length of a corrugation is (pitch) $\mathrm{Pt}=7 \mathrm{~mm}$. The depth of the cavity is $H=2.7 \mathrm{~mm}$. Since the cavity width is changing continuously with the cavity depth, width is determined at the mid-depth of the cavity ${ }^{18}$ as $W=5 \mathrm{~mm}$. The radius of the curvature for the edges inside the cavity is $r_{\text {up-(in) }}=r_{\text {down-(in) }}=1 \mathrm{~mm}$. The radius of the curvature for the edges at the cavity mouth is $r_{\text {up-(out) }}$ $=r_{\text {down }-(\text { out })}=0.5 \mathrm{~mm}$. The inner diameter is $D_{\text {in }}=26.5 \mathrm{~mm}$.

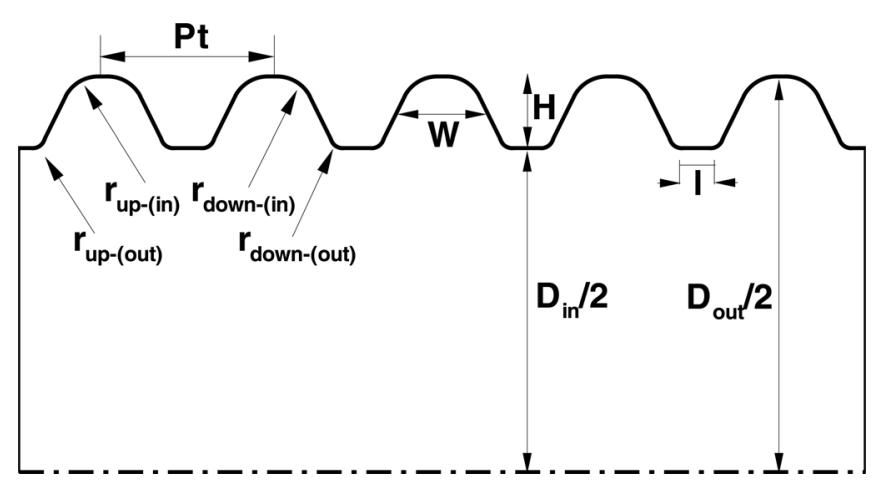

FIG. 3. Cross-section of a segment of Hummer with geometric parameters.

The plateau, which is the length of the constant inner diameter part between two cavities, is $l=1 \mathrm{~mm}$.

\section{Results}

\section{Average velocity profile and turbulence intensity}

All the velocity profiles that are presented were measured along an axis normal to the axis of Hummer at a distance of $1 \mathrm{~mm}$ downstream from the pipe termination. Some measurements were also taken inside the corrugated pipe, the results are identical with the presented data. It is convenient to measure the profile outside the pipe, because when the probe is in the pipe it is difficult to make measurements close to the wall.

In Fig. 4 a measured velocity profile for a straight Hummer is presented together with a turbulent velocity profile for a smooth pipe and a profile that is obtained by Reynolds-averaged Navier-Stokes (RANS) simulation of the Hummer. The velocity profile that is developed in the Hummer is rather different than the one of a smooth pipe. It is also seen that the RANS simulations can provide a reasonable estimation of the velocity profile.

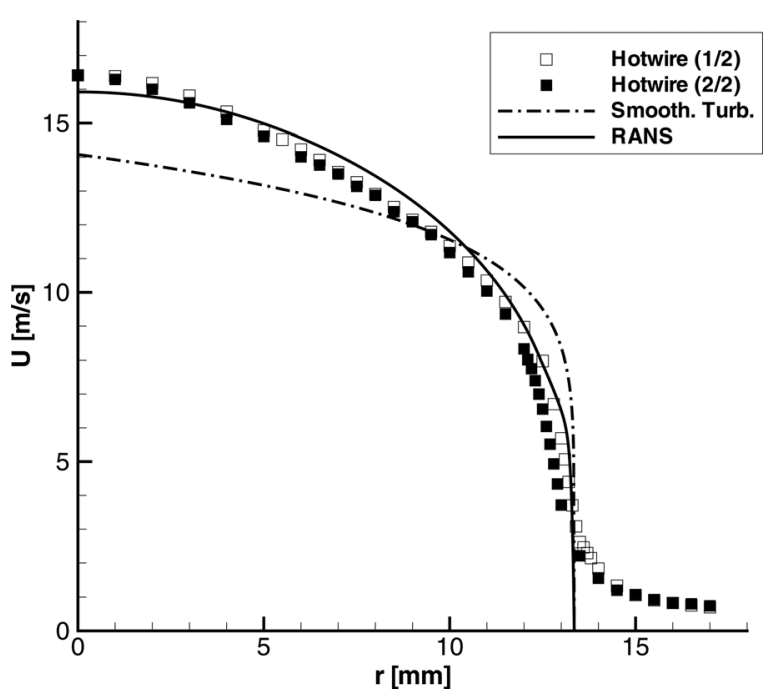

FIG. 4. Measured velocity profiles $U(r)$ for a Hummer: Hotwire(1/2), Hotwire(2/2) are the 1 st and 2 nd half of the profile, respectively. "Smooth. turb." indicates a fully turbulent pipe profile for a smooth pipe. RANS indicates a profile that is obtained by RANS simulation of a Hummer. 
The RANS simulations were performed with the commercial finite volume code FLUENT 6.3.

The computational domain had the same geometry as the Hummer but was composed of five cavities, as shown in Fig. 3. A cylindrical symmetric $2 \mathrm{D}$ domain was used to mimic a circumferential cavity. The computational domain contained approximately 180000 cells, which were clustered close to the cavity mouth where there are high gradients of velocity due to the shear layer. The pressure-based segregated solution algorithm SIMPLE (Ref. 19) was employed. A second-order upwind space discretization was used for convective terms. A $k-\epsilon$ turbulence model was used together with standard wall functions as near wall-treatment. The iterations were terminated when all residuals had dropped at least eight orders of magnitude. In the first simulation a fully developed turbulent velocity profile for a smooth pipe was used as an inlet boundary condition. Then the converged velocity profile at the outlet was extracted and used as the inlet velocity profile for the next simulation. This procedure was repeated until a fully developed velocity profile was obtained, namely 11 times, such that the imposed inlet velocity profile remained unaltered till the outlet. Thus, it took 50 corrugations for the flow to fully develop.

In Fig. 5, the measured turbulence intensity $\left(T I=u_{h}^{\prime} /\right.$ $\left.U_{\text {avrg }} \times 100\right)$ profile for a straight Hummer is presented. This rather high turbulence level hides the acoustic perturbations $u^{\prime}$ under the broadband hydrodynamic perturbations $\left(u_{h}^{\prime}\right)$ in a signal in the time domain.

The dimensionless fluctuation/perturbation amplitude, $\left|p_{\max }^{\prime}\right| / \rho_{0} c_{0} U_{\mathrm{avrg}}=\left|u_{\max }^{\prime}\right| / U_{\mathrm{avrg}}$, is defined as the amplitude of the standing pressure wave at a pressure anti-node inside the main pipe $\left|p_{\max }^{\prime}\right|$, divided by the air density $\rho_{0}$, the speed of sound $c_{0}$, and the average flow velocity $U_{\text {avrg; }}$; which is equal to the amplitude of acoustic velocity at a pressure node inside the main pipe $\left|u_{\max }^{\prime}\right|$ divided by the average flow

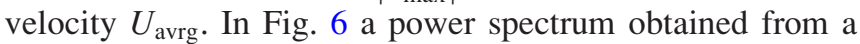
typical hotwire measurement is presented. In the Fourier domain the whistling frequency can easily be identified among the broadband hydrodynamic perturbations by the distinct peak in the spectrum. The corresponding perturbation amplitude $\left(\left|u_{\max }^{\prime}\right| / U_{\text {avrg }}\right)$ is determined as follows:

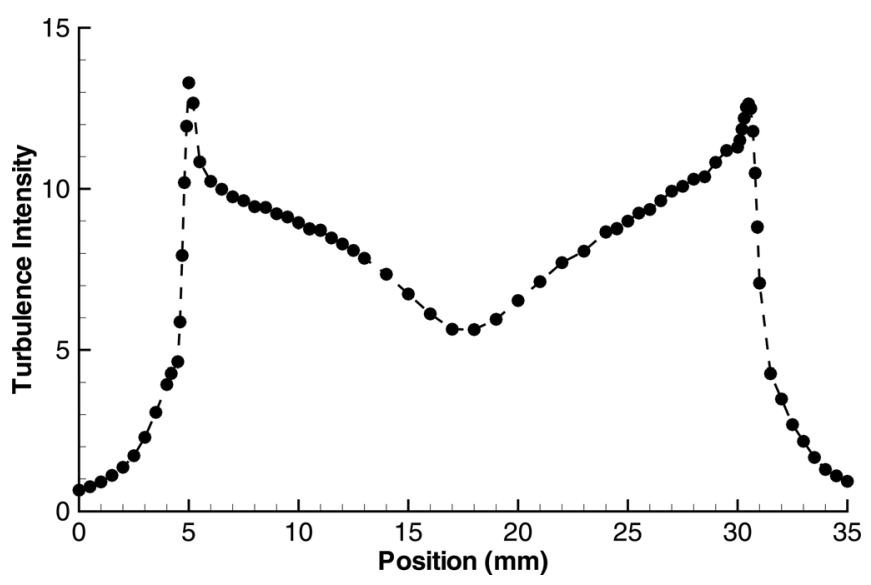

FIG. 5. Measured turbulence intensity profiles $\left(\mathrm{TI}=u_{h}^{\prime} / U_{\text {avrg }} \times 100\right)$ for a Hummer.

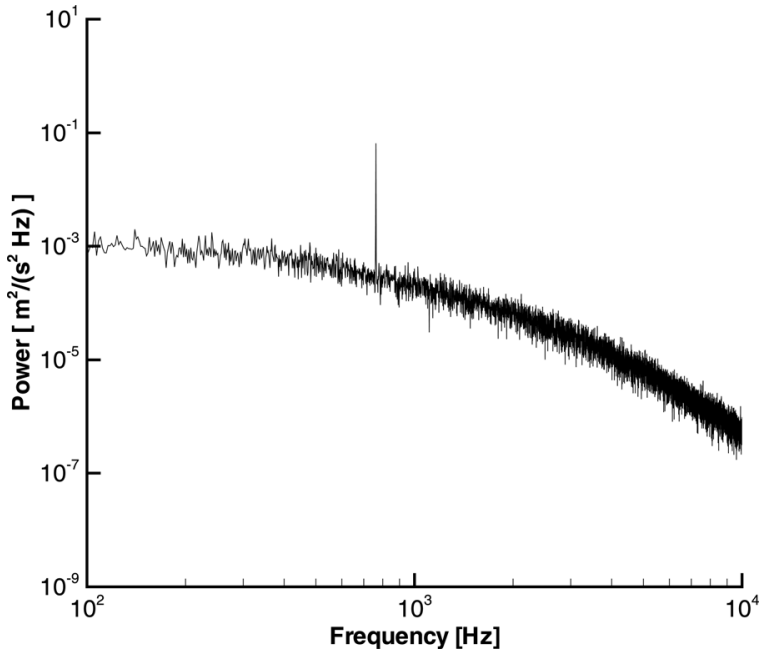

FIG. 6. A typical power spectrum $P(f)$ of a hotwire measurement for a whistling Hummer.

$$
\frac{\left|u_{\max }^{\prime}\right|}{U_{\mathrm{avrg}}}=\frac{\sqrt{\int P(f) d f}}{U_{\mathrm{avrg}}},
$$

where $P(f)$ is the power density.

\section{Friction factor}

The frictional pressure loss along a pipe of length $(L)$ is defined by

$$
p(0)-p(L)=4 c_{f} \frac{1}{2} \rho_{0} U_{\text {avrg }}^{2} \frac{L}{D_{\text {in }}},
$$

where $c_{f}$ is the friction factor. ${ }^{20}$ Measuring the settling chamber pressure $\left(p_{0}\right)$ as a function of average flow velocity $U_{\text {avrg }}$, the friction factor is determined as $c_{f}=1.78 \times 10^{-2}$ independent of the Reynolds number for $8 \times 10^{8} \leq \operatorname{Re}_{D}$ $\leq 4 \times 10^{4} \quad\left(\operatorname{Re}_{D}=U_{\mathrm{avrg}} D / \nu\right.$ with $\left.\nu=1.5 \times 10^{-5}\right)$. The pressure at the pipe inlet, $p(0)$, is calculated from the settling chamber pressure $p_{0}$ as follows $p(0)=p_{0}+1 / 2 \rho U_{\text {avrg }}^{2}$.

Knowing the friction factor $c_{f}$, a better estimation of the average flow velocity $\left(U_{\text {avrg }}\right)$ can be proposed than the frictionless model as

$$
U_{\mathrm{avrg}}=\frac{\Omega R}{\sqrt{1+4 c_{f} \frac{L}{D_{\mathrm{in}}}}},
$$

where $R$ is the rotation radius. As is explained in Sec. V A, $R \leq L$.

\section{Effective speed of sound}

The acoustic field in a Hummer, in a first order approximation, can be described in terms of plane waves propagating along the pipe axis. Sound propagates along the Hummer at an effective speed of sound ${ }^{18} c_{\text {eff }}$, which is lower than the speed of sound in the air $c_{0}$. As a first order approximation, the Hummer can be described as a tube of uniform cross section with a diameter of $D_{\text {in }}$. The inertia is determined 
considering the mass in this tube. The air in the cavities has a limited contribution to the inertia, ${ }^{21}$ however, they behave like an extra volume of air, which has the effect of lowering the frequency of each resonance. Thus, the acoustic compliance is determined by the total volume of the Hummer. Then, for the propagation of low frequency acoustic waves along the tube, $f \mathrm{Pt} / c_{0} \ll 1$, the effective speed of sound is estimated as follows:

$$
c_{\text {eff }}=c_{0} \sqrt{\frac{V_{\text {in }}}{V_{\text {tot }}}},
$$

where $V_{\text {in }}=\pi D_{\text {in }}^{2} / 4 L$ is the inner volume of the Hummer and $V_{\text {tot }}$ is the total volume of the Hummer. To determine the total volume of the Hummer, a section composed of 20 pitches $(140 \mathrm{~mm})$ was cut and one of the termination was closed by gluing it to a plastic plate. Using a syringe, starting from the bottom the tube was slowly filled to avoid air bubble formation. Then the difference in the weight of the empty corrugated segment and the corrugated segment filled with water was measured by means of a balance with an accuracy of $0.01 \mathrm{~g}$. The ratio of inner volume to total volume is found as $V_{\text {in }} / V_{\text {tot }}=0.83$, which leads to an effective speed of sound $c_{\text {eff }}=310 \mathrm{~m} / \mathrm{s}$ at room temperature $\left(c_{0}=340 \mathrm{~m} / \mathrm{s}\right)$.

\section{Whistling frequencies and Strouhal number}

In corrugated pipes the whistling frequency does not vary continuously with a monotonically increasing flow rate, but rather in distinct steps, corresponding to open-open resonant acoustic longitudinal modes of the pipe:

$$
f_{n}=\frac{n c_{\mathrm{eff}}}{2 L_{\mathrm{eff}}}, n=1,2,3, \ldots
$$

where $L_{\text {eff }}$ is the effective length of the pipe. Considering the experimental setup presented in Fig. 2, $L_{\text {eff }}$ corresponds to the combined length of the following elements: corrugated segment of the Hummer, the smooth segment of the Hummer, the connection piece to the wind tunnel, and the end corrections. ${ }^{21,22}$ Knowing the effective speed of sound and the whistling frequency of a given mode from an experiment, using Eq. $10 L_{\text {eff }}$ is determined as $822 \mathrm{~mm}$.

In Fig. 7 whistling frequencies, obtained from spectra as demonstrated in Fig. 6, in terms of Helmholtz number (He $=f L_{\text {eff }} / c_{\text {eff }}$ ), are given as a function of Mach number ( $\left.M=U_{\mathrm{avrg}} / c_{0}\right)$ for the Hummer. Integer and half integer values of Helmholtz number correspond to the even and odd longitudinal resonant modes, respectively. There is a global linear relationship between Helmholtz number and Mach number, which indicates a constant Strouhal number

$$
\mathrm{Sr}_{L_{c}}=f L_{c} / U_{\mathrm{avrg}}
$$

where $L_{c}$ is the characteristic length. Experiments have shown that the sum of the cavity width and the upstream edge radius $\left(L_{c}=W+r_{\text {up-(out) }}\right)$ is the most suitable characteristic length for Strouhal number. ${ }^{1,8}$ The Strouhal number is determined as $\mathrm{Sr}_{W+r_{\text {up }}}=0.44$ for the Hummer. Furthermore, it is seen that above a critical Mach number, $M=0.085$, a mode with a dif-

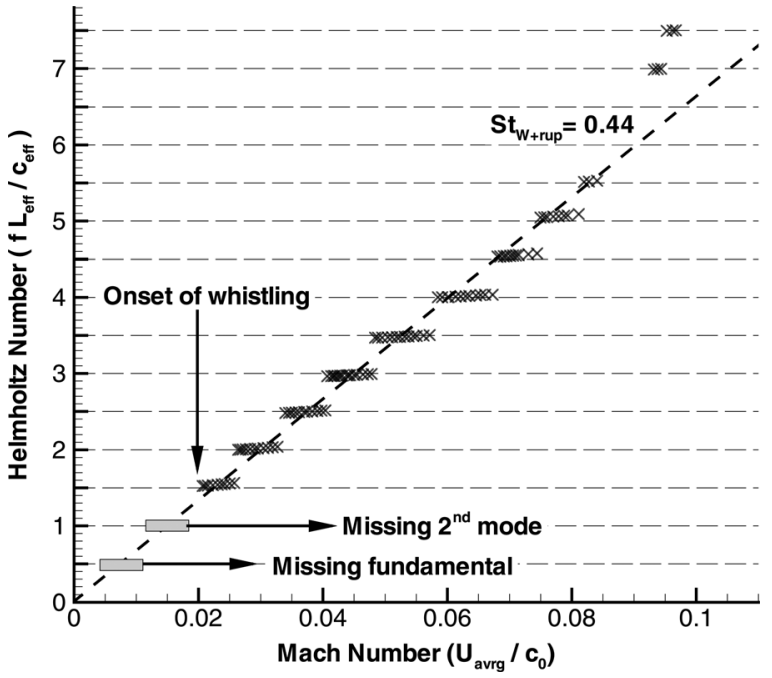

FIG. 7. Helmholtz number $\left(\mathrm{He}=f L_{\mathrm{eff}} / c_{\mathrm{eff}}\right)$ plotted against Mach number $\left(M=U_{\mathrm{avrg}} / c_{0}\right)$.

ferent Strouhal number is excited. The first mode above the critical Mach number corresponds to the first transversal pipe mode, $\mathrm{He}=f L_{\text {eff }} / c_{\text {eff }}=7$, based on the outer diameter $D_{\text {out }}$. This study is limited to the velocities below the critical Mach number.

The coupling of the flow instability at each cavity to the longitudinal standing wave can be described as a feedback loop which leads to self-sustained oscillations. In selfsustained oscillations the flow perturbations should undergo a total phase shift, when traveling along the feedback loop, matching an integer number of $2 \pi$. The total phase shift is mainly composed of a phase shift due to the convection of vortices from the upstream edge toward the downstream edge and due to the acoustical response of the pipe. The convection time of the vortices over the cavity mouth is $\left(W+r_{\text {up }}\right) / U_{\text {conv }}$ where the convection velocity is about half the main flow velocity $U_{\text {conv }}=U_{\text {avrg}} / 2$. Around a pipe resonance there is a rapid change in the phase of the acoustical response with a maximum of $\pi$ (change of sign). When the flow velocity in the pipe is increased the convection time of the vortices decreases so that the system increases the oscillation frequency $f$ to match the phase oscillation condition. ${ }^{16}$ The slope $d f / d U_{\text {avrg }}$ is inversely proportional to the quality factor of the resonator. If the quality factor of the resonator is large a small change in frequency is sufficient to provide a large acoustical contribution to the compensation of the convective phase shift. A closer look at the Fig. 7 reveals this feature. There is a slight, but discernible, increase in the whistling frequency within each resonant pipe mode $\left(f_{n}\right)$. Since the increase in the velocity is large compared to the corresponding increase in the frequency within the same acoustic resonant mode there is a range of Strouhal numbers, where the whistling is observed rather than a fixed Strouhal number. ${ }^{8,23,24}$ However, the response of the resonator has a maximum at the passive resonance frequency $f_{p}$ and therefore a maximum of the whistling amplitude at $f=f_{p}$. At this point the convection time of the vortices is close to a multiple of an oscillation period $T$ plus a quarter $(m+1 / 4) T=(m+1 / 4) / f(m=1,2,3, \ldots) .{ }^{25}$ This is further discussed in Sec. III C 6. 
In Fig. 8 normalized Helmholtz number $[\mathrm{He}=$ 2f $\left.L_{\text {eff }} /\left(n c_{\text {eff }}\right)\right]\left(\left|u_{\max }^{\prime}\right| / U_{\text {avrg }}\right)$ is plotted against Strouhal number $\left(\mathrm{Sr}_{W+r_{\text {up }}}\right)$ for acoustic modes of $3 \mathrm{rd}-11$ th. It is seen that using the effective speed of sound, $c_{\text {eff }}$, definition ${ }^{18}$ the whistling frequencies $\left(f_{n}\right)$ in a corrugated pipe can be predicted within $4 \%$.

\section{Onset of the Whistling}

The onset of the whistling in corrugated pipes has been observed at different longitudinal modes in the literature. In most of the studies the onset of whistling has been detected at the second acoustic longitudinal mode. ${ }^{3,4,26}$ Kristiansen and Wiik $^{27}$ have recorded an excited fundamental mode. Elliott ${ }^{18}$ obtained the whistling first for the ninth mode. In the current study, the third mode $(\mathrm{He}=1.5)$ is recorded as the first whistling mode. It is suggested in the literature that turbulence triggers the whistling. ${ }^{3,28,29}$ The absence of whistling at the fundamental mode is explained by the absence of turbulence.

At high flow rates the velocity field inside the pipe can display a complex unsteady chaotic motion called turbulence. The transition from a laminar (smooth-stationary) velocity field toward a turbulent (chaotic) flow is determined by the ratio of inertial to viscous forces. A measure for this is the Reynolds number. For a smooth pipe below $\operatorname{Re}_{D}=2300$ turbulence cannot be maintained. Depending on the inflow conditions, a laminar flow can, however, be maintained in a smooth pipe up to very high values of $\mathrm{Re}_{D} \cdot{ }^{20}$ In the case of rough walls (such as for a corrugated pipe) turbulence is commonly observed for $\operatorname{Re}_{D} \geq 4000$. $^{20}$ Transition can occur for $\mathrm{Re}_{D} \geq 2300$.

In Fig. 7 the expected Mach number ranges are indicated for the fundamental and the second mode, if they had been observed. The fundamental and second mode, would start whistling at $M=0.004\left(\operatorname{Re}_{D} \approx 2400\right)$ and $M=0.012$ $\left(\operatorname{Re}_{D} \approx 7200\right)$, respectively. The second mode corresponds to a fully turbulent flow, however it still does not sound. This experiment indicates that the absence of turbulence is

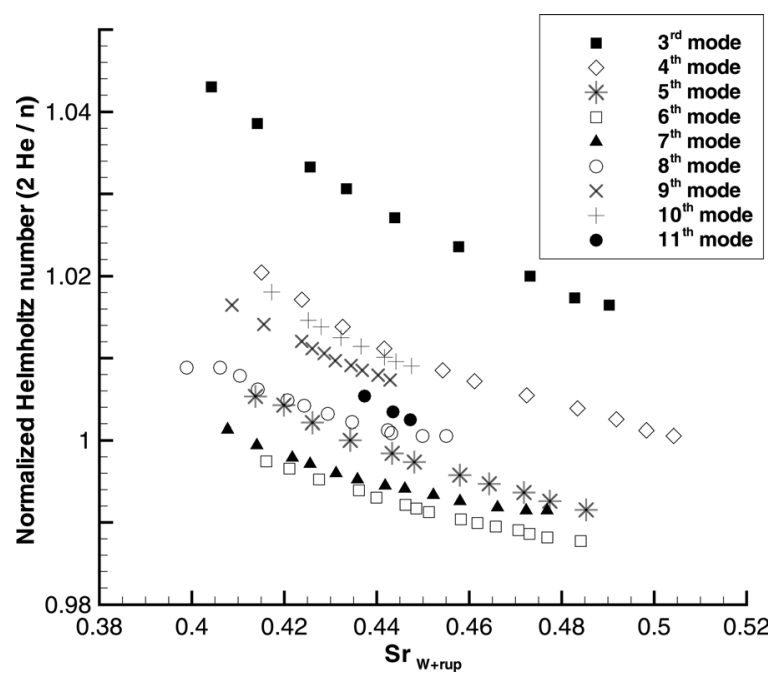

FIG. 8. Normalized Helmholtz number $\left(\mathrm{He}=2 f L_{\text {eff }} /\left(n c_{\text {eff }}\right)\right)\left(\left|u_{\max }^{\prime}\right| / U_{\text {avrg }}\right)$ plotted against Strouhal number ( $\mathrm{Sr}_{W+\text { rup }}$ ) for acoustic modes of $3 \mathrm{rd}-11$ th. not likely to be the essential factor determining whether a mode does not whistle. As it is explained later in Secs. IV B-IV D, turbulence has an effect on the whistling through its affect on the average velocity profile.

\section{Peak-whistling Strouhal number and whistling amplitude}

In Fig. 9 the perturbation amplitude $\left|u_{\max }^{\prime}\right| / U_{\text {avrg }}$ is plotted against the Strouhal number for all the whistling modes (3rd-11th). It is seen that all the modes appear in a narrow Strouhal number range between $0.4 \leq \mathrm{Sr}_{W+r_{\text {up }}} \leq 0.5$. The highest Strouhal number for a resonant mode indicates the onset of oscillations for that particular acoustic mode. It is called the critical Strouhal number ${ }^{30}\left(\mathrm{Sr}_{W+r_{\text {up }}}^{\mathrm{cr}}\right)$. It is also seen from Fig. 9 that within the same resonant mode after the onset of resonance, increasing the flow velocity increases the amplitude of pressure oscillations until reaches a peak value. Further increase of the flow velocity decreases the amplitude of pressure fluctuations. The Strouhal number, which corresponds to the maximum pressure fluctuation amplitude for a given acoustical mode, is called the peak-whistling Strouhal number ${ }^{7,12}$ $\left(\mathrm{Sr}_{W+r_{\text {up }}}^{\mathrm{p}-\mathrm{w}}\right)$. The peak-whistling Strouhal number of a corrugated pipe is determined through a linear least square fit of consecutive excited acoustic modes. ${ }^{8}$ The peak-whistling Strouhal number of the Hummer is determined to be $\mathrm{St}_{W+r_{\text {up }}}^{\mathrm{p}-\mathrm{w}}=0.44$, which is actually presented as the Strouhal number $\mathrm{St}_{W+r_{\text {up }}}$ $=0.44$ in Fig. 7 .

Experiments performed on commercial corrugated pipes of various lengths have shown that there exist a saturation in dimensionless pressure fluctuation amplitude around $\left|p_{\max }^{\prime}\right| /$ $\rho_{0} c_{0} U \approx 0.1$ when the pipe length $\left(L_{p}\right)$ reaches $L_{p} / D_{\text {in }}$ of 100. Further increase of the pipe length does not change the amplitude of pressure fluctuations. ${ }^{7}$ The Hummer produces a perturbation amplitude of $\left|u_{\max }^{\prime}\right| / U_{\mathrm{avrg}} \leq 0.08$, which is lower than the observed saturation value. However,

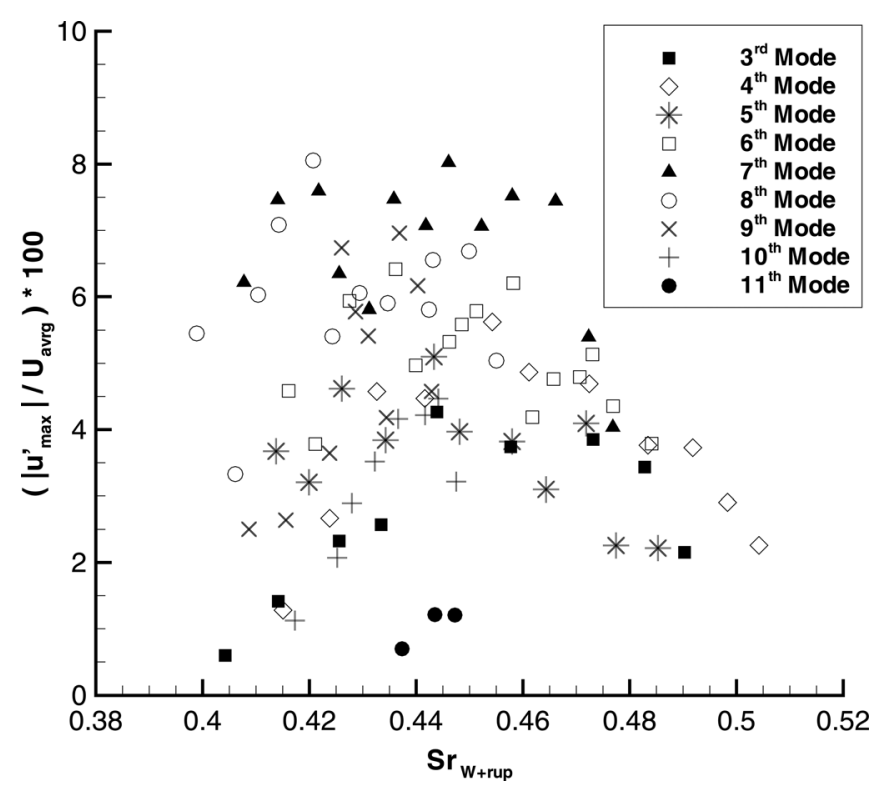

FIG. 9. Perturbation amplitude $\left(\left|u_{\max }^{\prime}\right| / U_{\text {avrg }}\right)$ plotted against Strouhal number $\left(\mathrm{Sr}_{W+\text { rup }}\right)$ for acoustic modes of $3 \mathrm{rd}-11$ th. 
considering the length of a Hummer $L_{p} / D_{\text {in }}=28$, it is reasonable that the observed perturbation amplitude is weaker.

\section{NUMERICAL METHODOLOGY}

In the previous study ${ }^{7}$ of the authors, a numerical methodology was proposed to investigate the aeroacoustic response of low Mach number confined flows to acoustic excitations. That study applied to corrugated pipes revealed the crucial importance of the velocity profile in the estimation of both the peak-whistling Strouhal number and the fluctuation amplitude. Experiments and RANS simulations carried out in the current study (Fig. 4), provide a better prediction of the flow profile in corrugated pipes. Thus, the proposed numerical methodology is revisited with more realistic flow profiles. In the first part of this section the methodology is briefly summarized and in the second part the improvements in the estimations are presented.

\section{A. An overview of the methodology}

The hydrodynamic instability, which is the driving force of the acoustic oscillations, is assumed to be a local phenomenon at each cavity. ${ }^{8,12}$ This implies that sound production is a local effect, which can be studied for a single cavity. Thus, one can try to describe the phenomenon by carrying out a numerical simulation of the flow within a single cavity instead of modeling the whole corrugated pipe. In this approach the possible hydrodynamic interactions between cavities are neglected and the oscillations are coupled through the longitudinal acoustical standing wave. Furthermore, knowing that pitch $P t$ is much smaller than the acoustic wavelength $c_{\text {eff }} / f_{n}$ of the produced sound wave, one can assume that wave propagation time is locally negligible. This corresponds to the assumption that the flow is locally incompressible. ${ }^{31}$

Following these ideas, incompressible 2D axisymmetric simulations were performed for a single cavity. The inlet of the computational domain is located at $0.5 \mathrm{~W}$ upstream of the cavity; such a short inlet pipe section is chosen to make sure that the imposed inlet velocity profiles do not evolve significantly before reaching the cavity. The outlet is placed at a reasonably far location, $9 \mathrm{~W}$ downstream, from the cavity. The computational domain contains approximately 70000 quadrilateral cells which are clustered close to the opening of the cavity and to the walls, where there are high gradients of velocity due to shear layer and boundary layer, respectively. A study on mesh dependence has been carried out. The same computation was performed with 2 times and 4 times more densely meshed domains, producing differences in the calculated acoustic source power of less than 5\%.

The simulations were carried out at low Reynolds numbers $(\operatorname{Re} \approx 4000)$ without turbulence modeling. The oscillating pressure differences $\Delta p^{\prime}$ induced along the pipe by the cavity oscillation are extracted from these simulations. At the inlet a uniform acoustic oscillating velocity in the axial direction $u^{\prime}$ is imposed in addition to the time averaged inlet velocity profile $U(r)$. As the viscous effects are not accurately described, the simulations are corrected by subtracting the pressure differences $\Delta p_{\text {visc }}^{\prime}$ obtained from simulations of the flow in a uniform pipe segment with the same boundary conditions as the cavity simulation. This correction can be interpreted as an extrapolation method for high Reynolds number flows, where the solution becomes Reynolds number independent. ${ }^{7}$ The acoustic power produced by the source is calculated as follows:

$$
P_{\text {source }}=S_{p} u^{\prime}\left(\Delta p^{\prime}-\Delta p_{\text {visc }}^{\prime}\right),
$$

where $S_{p}$ is the cross-sectional area. Finally, by taking the time average $\langle\cdots\rangle$ of the calculated acoustic energy $P_{\text {source }}$ over a sufficient number of oscillation periods, the spurious contribution due to the inertia is eliminated. ${ }^{7}$

\section{B. Effect of flow profile}

In the study of Martínez et al. ${ }^{31}$ on T-joints in pipe systems, a top hat velocity profile with a thin boundary layer was used as an inlet boundary condition. Later, Nakiboglu et $a l^{7}$ showed that a fully turbulent velocity profile of a smooth pipe is a better approximation for corrugated pipes. Experiments discussed in Sec. III C demonstrate that the turbulent velocity profile developed in a Hummer is noticeably different than that of smooth pipe (Fig. 4). Therefore, a series of RANS simulations was performed with a generic corrugated pipe geometry ${ }^{7}$ to obtain a more realistic velocity profile to employ as an inlet boundary condition. The parameters for the RANS simulations are the same as the ones used for the Hummer simulation. The geometric parameters of the generic corrugated pipe are as follows: $\mathrm{Pt}=2.25 W, \quad H=W, \quad D_{\text {in }}=4 W, \quad r_{\text {up }- \text { (out) }}=0.25 W$, $r_{\text {down-(out) }}=r_{\text {up-(in) }}=r_{\text {down-(in) }}=0$. These three velocity profiles, namely top hat pipe profile used by Martínez et al., ${ }^{31}$ fully turbulent pipe profile for a smooth pipe and profile that is obtained by RANS simulation of a generic corrugated pipe are compared in Fig. 10.

For a confinement ratio of $D_{\text {in }} /\left(W+r_{\text {up }}\right)=3.2$, in Fig. 11 estimated dimensionless average acoustic source power $\left\langle P_{\text {source }}\right\rangle /\left(\rho U_{\text {avrg }} S_{p}\left|u^{\prime}\right|^{2}\right)$ is presented as a function of Strouhal number for these velocity profiles. A negative $\left\langle P_{\text {source }}\right\rangle$ indicates that in that range of Strouhal numbers $\left(\mathrm{Sr}_{W+r_{\text {up }}}\right)$ the cavities act as acoustic sinks, which suppress the whistling. A positive $\left\langle P_{\text {source }}\right\rangle$ indicates that the cavities act as acoustic sources, which is a necessary condition for whistling. Here two ranges of Strouhal numbers $\left(\mathrm{Sr}_{W+r_{\text {up }}}\right)$ are observed for which $\left\langle P_{\text {source }}\right\rangle$ is positive. The lower $\left(0.4<S r_{W+r_{\text {up }}}<0.8\right)$ and the higher $\left(0.8<S r_{W+r_{\text {up }}}<1.4\right)$ Strouhal number ranges with positive average acoustic source power correspond to the second and the third hydrodynamic modes, respectively. In the second hydrodynamic mode there exist two vortices in the cavity mouth and the traveling time of the vortex across the opening is 1.25 oscillation period. Whereas for the third hydrodynamic mode three vortices are present at the same moment in the cavity mouth and a vortex takes 2.25 oscillation period to travel across the cavity. ${ }^{25}$ Experimentally observed Strouhal numbers (Fig. 9) correspond to the second hydrodynamic mode. It is clear that the peak-whistling Strouhal number, where the highest acoustic source power is registered, depends strongly on the velocity profile. With 


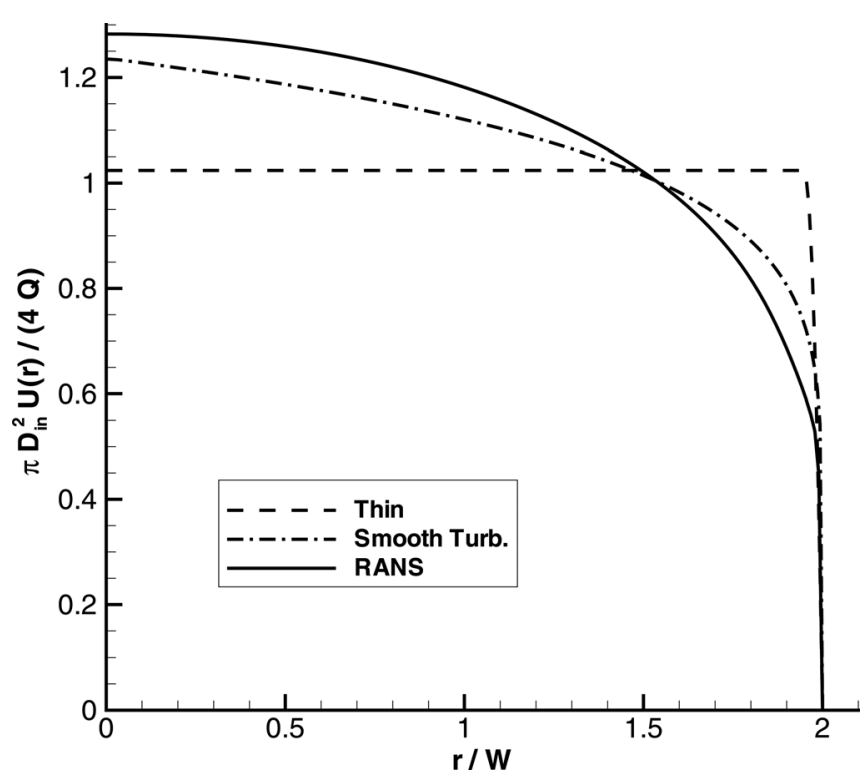

FIG. 10. Velocity profiles that are used as inlet boundary conditions. Thin indicates the thin turbulent pipe profile used by Martínez-Lera et al. (Ref. 31). "Smooth turb." indicates a fully turbulent pipe profile for a smooth pipe. RANS indicates a profile that is obtained by RANS simulation of a generic corrugated pipe.

increasing boundary layer thickness, the peak-whistling Strouhal number shifts to lower Strouhal numbers.

\section{Estimation of peak-whistling Strouhal number}

Considering the experimental data on corrugated pipes, ${ }^{8,18,32}$ a correlation between confinement ratio $D_{\text {in }} /$ $\left(W+r_{\text {up }}\right)$ and the measured peak-whistling Strouhal number $\mathrm{Sr}_{\mathrm{p}-\mathrm{w}}$ has been proposed: ${ }^{7}$

$$
\mathrm{St}_{\mathrm{p}-\mathrm{w}}=0.58\left(\frac{D_{\text {in }}}{W+r_{\text {up }}}\right)^{-0.2} \text {. }
$$

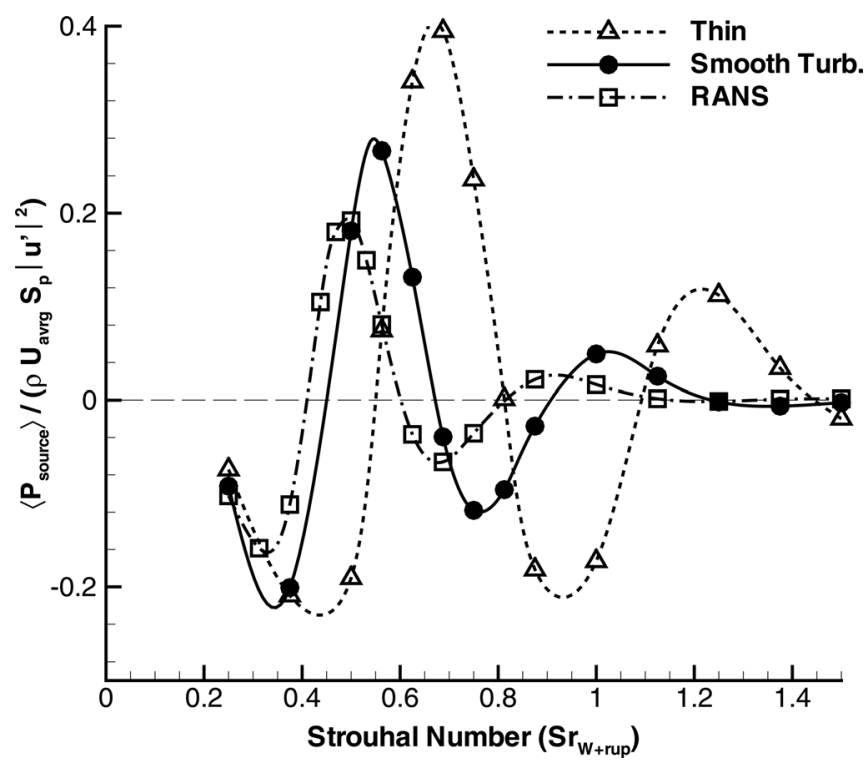

FIG. 11. Strouhal number plotted against dimensionless average acoustic source power $\left\langle P_{\text {source }}\right\rangle /\left(\rho U_{\mathrm{avrg}} S_{p}\left|u^{\prime}\right|^{2}\right)$ for a corrugated pipe with $D_{\text {in }} /$ $\left(W+r_{\text {up }}\right)=3.2$ and for a perturbation amplitude of $\left|u_{\max }^{\prime}\right| / U_{\text {avrg }}=0.05$ for the three velocity profiles given in Fig. 10 .
In Fig. 12 the proposed empiric formula [Eq. (13)] is presented. The peak-whistling Strouhal number obtained with the Hummer (Fig. 9) also follows this trend.

In an earlier study by the authors, ${ }^{7}$ using the numerical methodology summarized in Sec. IV A with a fully turbulent velocity profile of a smooth pipe (Fig. 10 - "smooth turb."), the peak-whistling Strouhal numbers were over-estimated by $10 \%$ as shown in Fig. 12. As demonstrated in Sec. III C 1, by performing RANS simulation of a corrugated geometry, a better estimation of the measured velocity profile can be obtained compared to a profile of a fully turbulent smooth pipe. Using the average velocity profile obtained from the RANS simulation of a generic corrugated pipe (Fig. 10- "RANS"), the same simulations have been repeated in this study. The predicted peak-whistling Strouhal number as a function of confinement ratio $D_{\text {in }} /\left(W+r_{\text {up }}\right)$ is also shown in Fig. 12. The numerical model predicts the peak-whistling Strouhal number within an accuracy of $2 \%$. It is evident that by using a more realistic flow profile, the numerical methodology produce much better estimations of the peak-whistling Strouhal number. This excellent agreement between the experiments and the numerical model confirms the significance of the effect of mean flow profile on the whistling behavior.

\section{Estimation of whistling amplitude in a long corrugated pipe}

In Fig. 13 estimated normalized dimensionless average acoustic source power $\left(D_{\text {in }} /\left(W+r_{\text {up }}\right)\right)\left\langle P_{\text {source }}\right\rangle /$ $\left(\rho U_{\mathrm{avrg}} S_{p}\left|u^{\prime}\right|^{2}\right)$ for a single corrugation is given as a function of perturbation amplitude $\left|u_{\max }^{\prime}\right| / U_{\text {avrg }}$ for the three different average velocity profiles given in Fig. 10 for a single cavity. The simulations were performed at respective peakwhistling Strouhal number of each profile, namely,

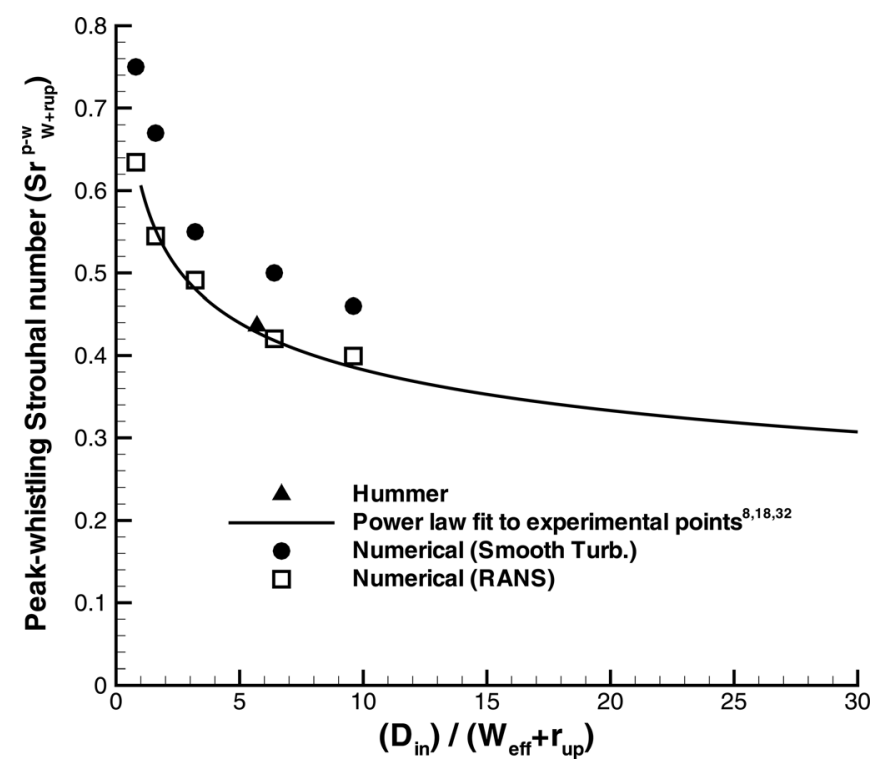

FIG. 12. Measured and estimated peak-whistling Strouhal numbers plotted against confinement ratio, $D_{\text {in }} /\left(W_{\text {eff }}+r_{\text {up }}\right)$. Power law fit [Eq. (13)] (Ref. 7) to the 18 experimental points obtained from three earlier studies (Refs. 8, 18,32 ). Data point of the Hummer. Numerical estimation by using two different velocity profiles given in Fig. 10: Numerical (smooth turb.) (Ref. 7) and numerical (RANS). 


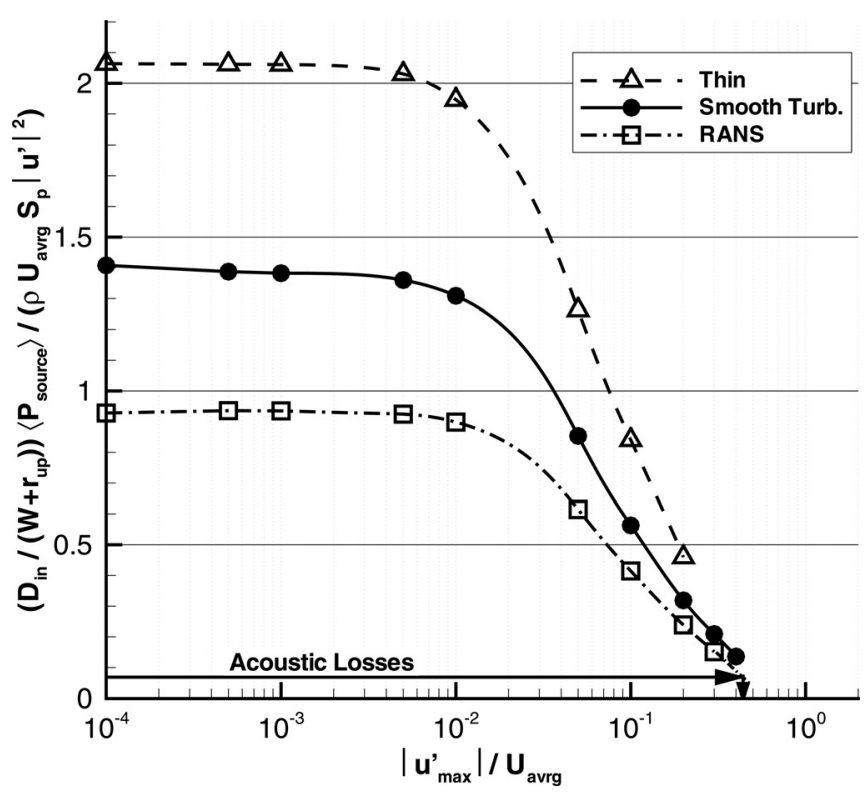

FIG. 13. Perturbation amplitude $\left|u_{\max }^{\prime}\right| / U_{\text {avrg }}$ is plotted against normalized dimensionless average acoustic source power $\left(D_{p} /\left(W+r_{\text {up }}\right)\right)\left\langle P_{\text {source }}\right\rangle /$ $\left(\rho U_{\text {avrg }} S_{p}\left|u^{\prime}\right|^{2}\right)$ for the 3 velocity profiles given in Fig. 10 . The confinement ratio is the same for all simulations $D_{\text {in }} /\left(W+r_{\text {up }}\right)=3.2$.

$\mathrm{St}_{W+r_{\text {up }}}^{\mathrm{p}-\mathrm{w}}=0.65$ for thin, $\mathrm{St}_{W+r_{\text {up }}}^{\mathrm{p}-\mathrm{w}}=0.55$ for smooth turb. and $S t_{W+r_{\text {up }}}^{p-w_{\text {up }}}=0.50$ for RANS.

It is seen that for all the profiles $\left|u_{\max }^{\prime}\right| / U_{\text {avrg }} \approx 5 \times 10^{-3}$ is the saturation point of the shear layer. For perturbations smaller than this the shear layer behaves linearly. Therefore, acoustic source power grows quadratically with $\left|u_{\max }^{\prime}\right| / U_{\mathrm{avrg}}$, making the dimensionless average acoustic source power $\left\langle P_{\text {source }}\right\rangle /\left(\rho U_{\mathrm{avrg}} S_{p}\left|u^{\prime}\right|^{2}\right)$ constant. Above the saturation point, nonlinearities become dominant and $\left\langle P_{\text {source }}\right\rangle /\left(\rho U_{\text {avrg }} S_{p}\left|u^{\prime}\right|^{2}\right)$ starts to decrease with $\left|u_{\max }^{\prime}\right| / U_{\text {avrg }}$.

To estimate the amplitude of the whistling in a long corrugated pipe an energy balance model is required. In a first order approximation radiation losses at the pipe terminations and convective losses due to vortex shedding are small compared to the visco-thermal losses and can be neglected in a long corrugated pipe. Then the energy balance is simplified to

$$
\frac{2}{\pi}\left\langle P_{\text {source }}\right\rangle=\left\langle P_{\text {visc }}\right\rangle
$$

where $\left\langle P_{\text {source }}\right\rangle$ is the time averaged acoustic source power and $\left\langle P_{\text {visc }}\right\rangle$ is the time averaged power loss due to viscothermal losses, which is estimated for a single cavity as follows:

$$
\frac{\left\langle P_{\mathrm{visc}}\right\rangle}{\rho U_{\mathrm{avrg}} S_{p}\left|u_{\max }^{\prime}\right|^{2}}=\frac{1}{2} \frac{c_{\mathrm{eff}} \alpha P t}{U_{\mathrm{avrg}}} .
$$

The factor $(2 / \pi)$ in Eq. (14) takes into account the spatial dependency of the acoustical velocity $\left(u^{\prime}\right)$ along a standing wave. $^{7}$ Assuming a quasisteady flow, ${ }^{33}$ the fluctuating pressure drop is stated as follows:

$$
\frac{d p^{\prime}}{d x}=\rho U_{\text {avrg }} u^{\prime} \frac{4 c_{f}}{D_{\text {in }}},
$$

where $c_{f}=1.78 \times 10^{-2}$ is the experimentally determined resistance coefficient and related to the damping coefficient for acoustic waves by

$$
\alpha=\frac{U_{\mathrm{avrg}}}{c_{\mathrm{eff}}} \frac{4 c_{f}}{D_{\mathrm{in}}} .
$$

Combining Eqs. (15)-(17), the normalized dimensionless visco-thermal losses is estimated as $(\pi / 2)\left(D_{\text {in }} /(W\right.$ $\left.\left.+r_{\text {up }}\right)\right)\left\langle P_{\text {visc }}\right\rangle /\left(\rho U_{\text {avrg }} S_{p}\left|u^{\prime}\right|^{2}\right)=\pi c_{f} P t /\left(W+r_{\text {up }}\right)=0.065$. Which leads to a maximum perturbation amplitude of $\left|u_{\max }^{\prime}\right| /$ $U_{\text {avrg }} \approx 0.45$ (Fig. 13). Considering the experimental data of $\left|u_{\max }^{\prime}\right| / U_{\text {avrg }}=0.1$, all the profiles lead to an overestimated value.

It should be noticed that this approach has a fundamental drawback. ${ }^{7}$ The losses due to flow separation at each cavity are implicitly included in the simulations. By introducing the experimentally measured resistance coefficient $\left(c_{f}\right)$ to calculate the damping coefficient, this non-linear effect is again taken into account in this approach. Also the model neglects heat transfer losses.

\section{RADIATION}

Up to now the flow inside the Hummer has been described. In this section the wave propagating from the open ends of the tube towards a listener is considered. First the theory is discussed, secondly the acoustic measurements are presented and in the last part the measured sound pressure levels are compared with the predictions from the theory.

\section{A. Theory}

The radiation from a Hummer can be modeled as a two pulsating spheres (monopoles) at the two open extremities of the tube. Depending on the acoustic mode (standing wave, $n=1,2,3, \ldots)$ they pulsate in phase or in opposite phase. The strength of these monopoles is estimated for a given acoustic mode as

$$
Q_{n}=u_{n}^{\prime} S_{p}=\frac{u_{n}^{\prime}}{U_{\mathrm{avrg}}} U_{\mathrm{avrg}} S_{p}=\frac{u_{n}^{\prime}}{U_{\mathrm{avrg}}} \frac{\Omega_{n} R}{\sqrt{1+4 c_{f} \frac{L}{D_{\mathrm{in}}}}} S_{p}
$$

where $u_{n}^{\prime} / U_{\mathrm{avrg}} \approx 0.05$ is determined from the measurements (Fig. 9) and the average velocity is estimated from Eq. (8). Here $S_{p}$ and $R$ are cross-sectional area and the radius of the rotation of the Hummer, respectively. As shown schematically in Fig. 14, because of the swinging motion the Hummer bends. Thus, the radius of the rotation of the Hummer is smaller than the length of the Hummer $(R \leq L)$. Knowing the Strouhal number from Fig. 9, the rotation speed $\Omega_{n}$ can be estimated as follows:

$$
\Omega_{n}=\frac{f_{n}\left(W+r_{\text {up }}\right)}{\operatorname{Sr}_{W+r_{\mathrm{up}}} R} \sqrt{1+4 c_{f} \frac{L}{D_{\mathrm{in}}}} .
$$

As indicated in Fig. 14 the location of the fixed monopole $\left(S_{1}\right)$, the hand hold side of the Hummer, is taken as the origin of the space $\vec{x}_{s 1}=(0,0,0)$. Then the location of the rotating 


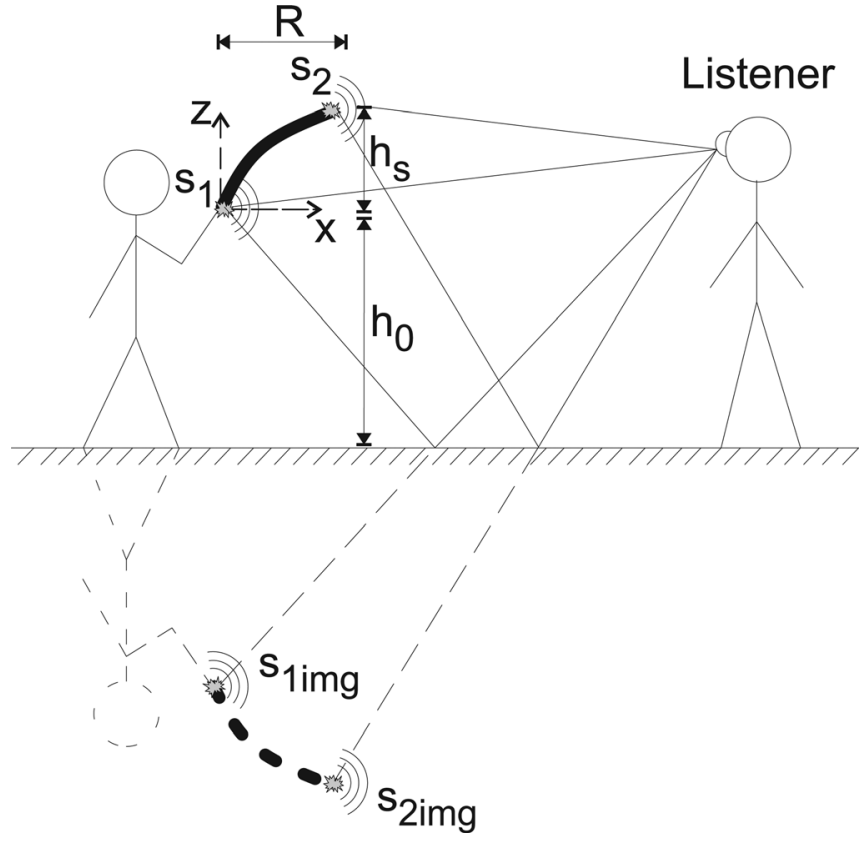

FIG. 14. The schematic drawing of a Hummer in action with a listener.

source $\left(S_{2}\right)$ is defined as $\vec{x}_{s 2}=\left(R \cos \left(\Omega_{n} t_{e}\right), R \sin \left(\Omega_{n} t_{e}\right), h_{s}\right)$. Here $t_{e}$ is the emission time of the rotating source, $S_{2}$, and it is related to the time $t$ at which the wave reaches the listener by

$$
t=t_{e}+\frac{\left|\vec{x}-\vec{x}_{s 2}\left(t_{e}\right)\right|}{c_{0}},
$$

and $h_{s}$ is the vertical distance between the rotating and fixed sources. Experiments have been performed in a semianechoic chamber, where the floor is reflecting. Reflections from the ground can be modeled by method of images. ${ }^{34}$ The listener will hear a superposition of the direct waves from sources $S_{1}, S_{2}$ and the reflected waves, coming from the image sources $S_{\text {1img }}, S_{2 \text { img. }}$. Using a quasi-steady approach, in which the position of the moving source $\left(S_{2}\right)$ is parametrized as a function of retarded time, the pressure field at the listener position can be calculated, using the complex notation with $e^{i \omega t}$ convention as ${ }^{34}$

$$
\begin{aligned}
\hat{p}\left(\vec{x}, \omega_{n}\right)= & \rho_{0} \frac{i \omega_{n} Q_{n}}{4 \pi}\left\{\left(\frac{e^{-i k_{n}\left|\vec{x}-\vec{x}_{s 1}\right|}}{\left|\vec{x}-\vec{x}_{s 1}\right|}+\frac{e^{-i k_{n}\left|\vec{x}-\vec{x}_{s \operatorname{limg}}\right|}}{\left|\vec{x}-\vec{x}_{s 1 \mathrm{img}}\right|}\right)\right. \\
& \left.+(-1)^{n+1}\left(\frac{e^{-i k_{n}\left|\vec{x}-\vec{x}_{s 2}\left(t_{e}\right)\right|}}{\left|\vec{x}-\vec{x}_{s 2}\left(t_{e}\right)\right|}+\frac{e^{-i k_{n}\left|\vec{x}-\vec{x}_{s 2 i m g}\left(t_{e}^{*}\right)\right|}}{\left|\vec{x}-\vec{x}_{s 2 \mathrm{img}}\left(t_{e}^{*}\right)\right|}\right)\right\},
\end{aligned}
$$

where $t_{e}^{*}$ is the retarded time of the rotating image source. Here the wave number is estimated as

$$
k_{n}=\frac{\omega_{n}}{c_{0}}=\frac{2 \pi f_{n}}{c_{0}}=\frac{\pi c_{e f f}}{c_{0} n L},
$$

where $n$ is the mode number, $c_{0}$ is the speed of sound, $c_{e f f}$ is the effective speed of sound [Eq. (9)] and $L$ is the length of the Hummer.

In the previous statement [Eq. (21)] for the pressure field at the listener position, the effect of the Doppler shift due to the rotating source is not incorporated. As explained by Dowling and Williams, ${ }^{35,36}$ the sound field including the Doppler shift that is generated by a moving monopole source is given by

$$
p^{\prime}(\vec{x}, t)=\rho_{0} \frac{\partial}{\partial t}\left(\frac{Q\left(t_{e}\right)}{4 \pi\left|\vec{x}-\vec{x}_{s}\left(t_{e}\right)\right|\left|1-M_{s}\left(t_{e}\right)\right|}\right),
$$

where $\vec{x}$ is the listener position, $t_{e}$ is the retarded time, $Q\left(t_{e}\right)$ is the source strength, and $c_{0} M_{s}\left(t_{e}\right)$ is the component of the source velocity in the direction of the observer. In the case of the Hummer, by a superposition of the four sources of sound, the following expression is obtained in the time domain:

$$
\begin{aligned}
p^{\prime}(\vec{x}, t)= & \frac{-\omega_{n} \rho_{0} Q_{n}}{4 \pi}\left(\frac{\sin \left(\omega_{n}\left(t-\frac{\left|\vec{x}-\vec{x}_{s 1}\right|}{c_{0}}\right)\right)}{\left|\vec{x}-\vec{x}_{s 1}\right|}+\frac{(-1)^{n+1} \sin \left(\omega_{n} t_{e}\right)}{\left(1-M_{s 2}\left(t_{e}\right)\right)^{2}\left|\vec{x}-\vec{x}_{s 2}\left(t_{e}\right)\right|}\right) \\
& +\left(\frac{\rho_{0} Q_{n}}{4 \pi} \frac{\left(\vec{x}-\vec{x}_{s 2}\left(t_{e}\right)\right) \frac{\vec{a}_{s 2}\left(t_{e}\right)}{c_{0}}+c_{0} M_{s 2}\left(t_{e}\right)-\frac{\left|\vec{v}_{s 2}\left(t_{e}\right)\right|^{2}}{c_{0}}}{\left|\vec{x}-\vec{x}_{s 2}\left(t_{e}\right)\right|^{2}\left(1-M_{s 2}\left(t_{e}\right)\right)^{3}}\right) \\
& +\frac{-\omega_{n} \rho_{0} Q_{n}}{4 \pi}\left(\frac{\sin \left(\omega_{n}\left(t-\frac{\left|\vec{x}-\vec{x}_{s 1 \mathrm{img}}\right|}{c_{0}}\right)\right)}{\left|\vec{x}-\vec{x}_{s 1 \mathrm{img}}\right|}+\frac{(-1)^{n+1} \sin \left(\omega_{n} t_{e}^{*}\right)}{\left(1-M_{s 2 \mathrm{img}}\left(t_{e}^{*}\right)\right)^{2}\left|\vec{x}-\vec{x}_{s 2 \mathrm{img}}\left(t_{e}^{*}\right)\right|}\right) \\
& +\left(\frac{\rho_{0} Q_{n}}{4 \pi} \frac{\left(\vec{x}-\vec{x}_{s 2 \mathrm{img}}\left(t_{e}^{*}\right)\right) \cdot \frac{\vec{a}_{s 2 \mathrm{img}}\left(t_{e}^{*}\right)}{c_{0}}+c_{0} M_{s 2 \mathrm{img}}\left(t_{e}^{*}\right)-\frac{\left|\vec{v}_{s 2 \mathrm{img}}\left(t_{e}^{*}\right)\right|^{2}}{c_{0}}}{\left|\vec{x}-\vec{x}_{s 2 \mathrm{img}}\left(t_{e}^{*}\right)\right|^{2}\left(1-M_{s 2 \mathrm{img}}\left(t_{e}^{*}\right)\right)^{3}}\right)
\end{aligned}
$$


where the following definitions are used for the velocity of the moving source and its image:

$$
\vec{v}_{s 2}\left(t_{e}\right)=\frac{\partial \vec{x}_{s 2}\left(t_{e}\right)}{\partial t_{e}}, \quad \vec{v}_{s 2 \mathrm{img}}\left(t_{e}^{*}\right)=\frac{\partial \vec{x}_{s 2 \mathrm{img}}\left(t_{e}^{*}\right)}{\partial t_{e}^{*}},
$$

the acceleration of the moving source and its image:

$$
\vec{a}_{s 2}\left(t_{e}\right)=\frac{\partial^{2} \vec{x}_{s 2}\left(t_{e}\right)}{\partial t_{e}^{2}}, \quad \vec{a}_{s 2 \mathrm{img}}\left(t_{e}^{*}\right)=\frac{\partial^{2} \vec{x}_{s 2 \mathrm{img}}\left(t_{e}^{*}\right)}{\partial t_{e}^{* 2}},
$$

and the Mach number of the moving source and its image:

$$
\begin{aligned}
& M_{s 2}\left(t_{e}\right)=\frac{\vec{x}-\vec{x}_{s 2}\left(t_{e}\right)}{\left|\vec{x}-\vec{x}_{s 2}\left(t_{e}\right)\right|} \cdot \frac{\vec{v}_{s 2}\left(t_{e}\right)}{c_{0}}, \\
& M_{s 2 \mathrm{img}}\left(t_{e}^{*}\right)=\frac{\vec{x}-\vec{x}_{s 2 \mathrm{img}}\left(t_{e}^{*}\right)}{\left|\vec{x}-\vec{x}_{s 2 \mathrm{img}}\left(t_{e}^{*}\right)\right|} \cdot \frac{\vec{v}_{s 2 \mathrm{img}}\left(t_{e}^{*}\right)}{c_{0}} .
\end{aligned}
$$

The first model [Eq. (21)], which does not incorporate the Doppler shift, is compared with the second model [Eq. (24)] in Fig. 15. Estimated pressure amplitudes from these two models $\hat{p}_{\text {wout }}$ and $\hat{p}_{\text {with }}$ for a listener position of $\vec{x}=(0.8 m, 0,0)$ are given as a function of time for a single period of rotation for the fifth mode $(n=5)$. The first model can capture all the amplitude modulations. As shown in the combined deviation plot the relative difference, $\left(\hat{p}_{\text {wout }}-\hat{p}_{\text {with }}\right) /\left(\hat{p}_{\text {with }}\right)_{\max } \times 100$, between the predicted amplitudes is less than $10 \%$. $\left(\hat{p}_{\text {with }}\right)_{\max }$ is the maximum of $\hat{p}_{\text {with }}$ over a rotation period.

In Fig. 16 estimated pressure amplitudes for listener positions of $\vec{x}=(0.8 m, 0,0)$ and $\vec{x}=(3 m, 0,0)$ are presented for two cases, where the reflections from the floor are included and not included. The calculations are performed for the third mode $(n=3)$ using the simple model [Eq. (21)]. It is seen that for the listener position of $\vec{x}=(0.8 m, 0,0)$ the effect of
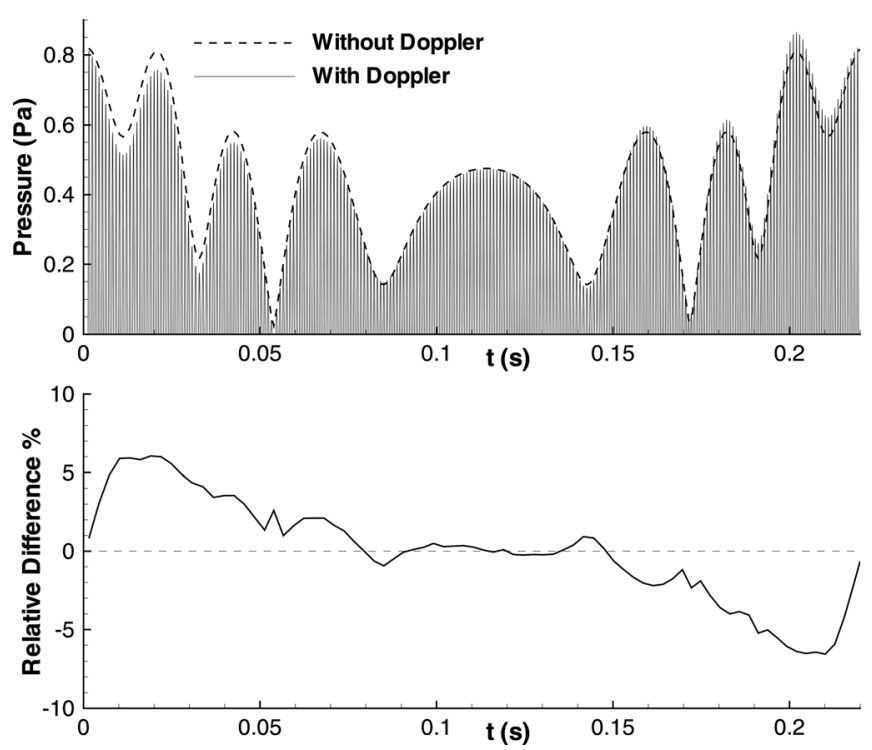

FIG. 15. Estimated pressure amplitudes for a listener position of $\vec{x}=(0.8 m, 0,0)$ for the fifth mode $n=5$ for a single period of rotation for two models: without Doppler shift $\left[\hat{p}_{\text {wout }}\right.$, Eq. (21)] and with Doppler shift $\left[\hat{p}_{\text {with }}\right.$, Eq. (24)]. Relative difference is $\left(\hat{p}_{\text {wout }}-\hat{p}_{\text {with }}\right) /\left(\hat{p}_{\text {with }}\right)_{\max } \times 100$. Radius of rotation is $R=0.8 L$.
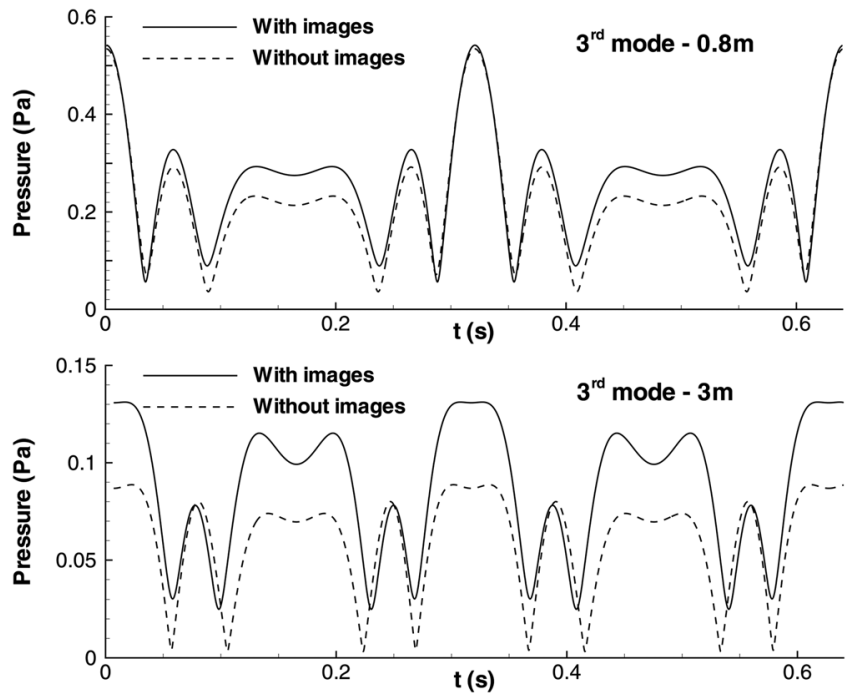

FIG. 16. Estimated pressure amplitudes at listener positions of $\vec{x}=(0.8 m, 0,0)$ and $\vec{x}=(3 m, 0,0)$ for the third mode $n=3$ for two periods of rotation: with (with images) and without (without images) taking the reflections from the floor into account.

the reflections on the amplitude is not pronounced. In particular, reflections have no effect on the maximum amplitude experienced by the listener at the moment when the Hummer reaches the closest position to the listener $(t=0, t=0.325)$. This is simply because the rotating source $\left(S_{2}\right)$ dominates all the other sources ( $S_{1}, S_{1 \mathrm{img}}$, and $\left.S_{2 \text { img }}\right)$ at such a close distance from the listener. At a listener position of $\vec{x}=(3 m, 0,0)$ the effect of the reflections is notable. Reflections increase the pressure amplitudes by $50 \%$ at the listener position. This is due to the fact that at such a listener position, the distance between the real sources $\left(S_{1}, S_{2}\right)$ and the listener becomes comparable to the distance between the imaginary sources $\left(S_{\text {limg }}, S_{2 \text { img }}\right)$ and the listener.

From Figs. 15 and 16 it is concluded that the Doppler shift does not have an essential role on the amplitude modulation. It is primarily controlled by the interference of the fixed $\left(S_{1}\right)$ and moving $\left(S_{2}\right)$ sources. Depending on the listener position the image sources $\left(S_{1 \mathrm{img}}, S_{2 \mathrm{img}}\right)$ can also have a very strong affect on the amplitude modulation.

There is an essential difference in the amplitude modulation mechanism between a Hummer and a Leslie horn. In a Leslie horn there exist only one monopole source. ${ }^{37}$ Thus, the amplitude modulation depends on the presence of reflections. ${ }^{38}$ In the Hummer, however, as discussed the reflections are not a necessary condition for the interference pattern.

\section{B. Experiments}

Experiments were performed in a semi-anechoic room with a reflecting floor. The chamber has a volume of $100 \mathrm{~m}^{3}$ and a cut-off frequency of $300 \mathrm{~Hz}$. As schematically shown in Fig. 14, the Hummer was played by swirling it in a circular motion above the head of the performer roughly keeping the moving termination in a horizontal plane. The sound pressure level was recorded by means of two microphones (Brüel \& Kjær type 4133 and 4165). One of the microphones was held by the Hummer player close to the pipe termination 
by holding the microphone and the Hummer together in the same hand. The microphone was placed against the tube $3 \mathrm{~cm}$ from the opening. This microphone will be referred as hand microphone. The second microphone was held by the listener at various distances from the performer, which will be referred as the distant microphone. The performer played approximately 15 seconds at each mode, while it was recorded simultaneously by the two microphones. The performer was also recorded by means of a video camera, which was used to estimate the rotation speed $\Omega_{n}$, the radius of the rotation $R$, and the vertical distance $h_{s}$ between the rotating and the fixed sources.

In Fig. 17 the signals obtained from the hand and the distant microphone are shown when the performer was playing the 3rd acoustic mode for a duration of two periods of rotation. The distant microphone was $0.8 \mathrm{~m}$ from the performer. The signal from the distant microphone shows a very strong amplitude modulation while the hand microphone displays a weak modulation. The amplitude modulations observed at the hand microphone is an indication of non-constant rotation velocity $\Omega_{n}$ during the performance. The amplitude modulation of the distant microphone is discussed in the next section.

In Fig. 18 the same signals (Fig. 17) are presented in the Fourier domain. The sound pressure level (SPL) recorded in the vicinity of the fixed source was around $115 \mathrm{~dB}$ and $70 \mathrm{~dB}$ at a distance of $0.8 \mathrm{~m}$ from the performer. It is clear that the spectrum is dominated by the fundamental oscillation frequency $f_{n}=637 \mathrm{~Hz}$, corresponding to the third acoustic mode $(n=3)$ and its exact multiples at $m f_{n}(m=1,2,3, \ldots)$. These higher harmonics are due to the non-linear saturation mechanism, which limits the amplitude of the oscillations ${ }^{16}$ (Sec. IV D).

In Fig. 19 frequency spectra are plotted against sound pressure levels for the 3rd and 5th acoustic modes around their respective fundamental oscillation frequencies (whistling) both for the hand and the distant microphones $(0.8 \mathrm{~m})$. An obvious difference between the pressure recorded by the
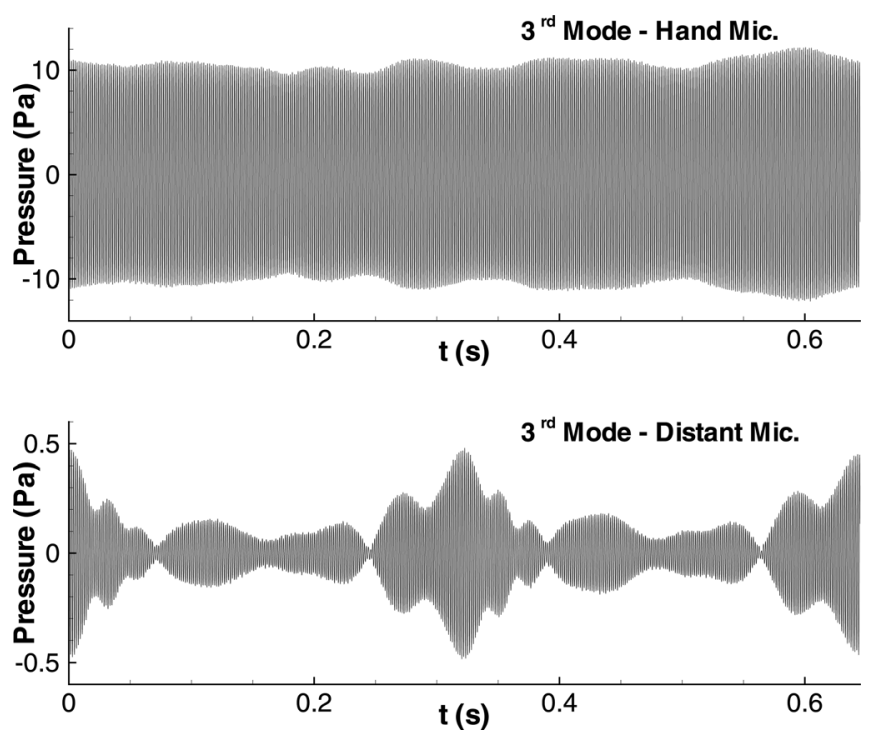

FIG. 17. Signals from the distant microphone $(0.8 \mathrm{~m})$ and the hand microphone for the 3 rd acoustic mode for two periods of rotation. hand microphone and the distant microphone is the width of the peaks in the spectrum. The signal from the hand microphone has a sharp peak. The signal from the distant microphone, however, has a rather broad peak. This is due to the Doppler shift and is also observed for the Leslie horn. ${ }^{37,38}$ During a rotation when the Hummer is moving toward the microphone it creates a side peak at a higher frequency than recorded at the hand microphone, and vice versa when it is moving away from the microphone. It is also evident that these two side peaks are not exactly symmetric with respect to the center peak, particularly for the 3rd mode, which indicates that the rotation velocity towards and away from the microphone is not the same. The width of the broad peaks are 40 and $120 \mathrm{~Hz}$ for the 3rd and 5th acoustic modes, respectively. The relative Doppler broadening reaches $\Delta f / f=6 \%$ which corresponds to half a tone. Therefore it is perceptually quite important.

\section{Comparison}

In this section the signals that are obtained from the experiments are compared with the estimated signals from the theory [Eq. (24)]. In Fig. 20 and Fig. 21 measured and estimated pressure amplitudes for the listener positions of $\vec{x}=(0.8 m, 0,0)$ and $\vec{x}=(3 m, 0,0)$ are given as a function of time during two periods of rotation for the modes of $n=3,4$, and 5, respectively. For ease of comparison the peaks in the pressure modulation are indexed.

First, it should be mentioned that for the same mode $n$ ) the period lengths of the signals are not the same for the listener positions of 0.8 and $3 \mathrm{~m}$. This is due to the fact that the signals were obtained from two different experiments. As demonstrated in Fig. 7, the whistling occurs for a range of velocity within a specific mode. Thus during the experiments, although the Hummer was whistling at the same mode, the rotation speeds $\left(\Omega_{n}\right)$ were not the exactly the same. Secondly, it should be noted that for the calculation of the signals from the theory, the rotation speeds $\left(\Omega_{n}\right)$ obtained from the respective experiments are used instead of the ones obtained from the theory [Eq. (19)]. Theory overestimates $\Omega_{n}$ by $\approx 30 \%$.

An apparent difference between the measured and the estimated signals is the lack of symmetry between the first and the second half of the rotation period. Due to the nonconstant rotation velocity $\left(\Omega_{n}\right)$ during the performance, recorded as small fluctuations in the signal from the hand microphone (Fig. 17), there exist an asymmetry between the first and the second half of the rotation period for all the measured signals.

The Hummer produces radiation patterns similar to the ones observed in flue organ pipes as explained by Fletcher and Rossing. ${ }^{16}$ This is due to the fact that $c_{\text {eff }} \leq c_{0}$, so that the two radiating monopoles are at a distance from each other smaller than $(n \lambda / 2)$, where $n$ is the acoustic longitudinal mode and $\lambda$ is the wave length. In organ pipes the same effect (end correction) is due to the inertia of the flow through the pipe mouth. ${ }^{16}$ During the performance the Hummer creates an amplitude modulation at a listener position due to the rotation of these radiation patterns. It is seen that the estimated signals from the theory 


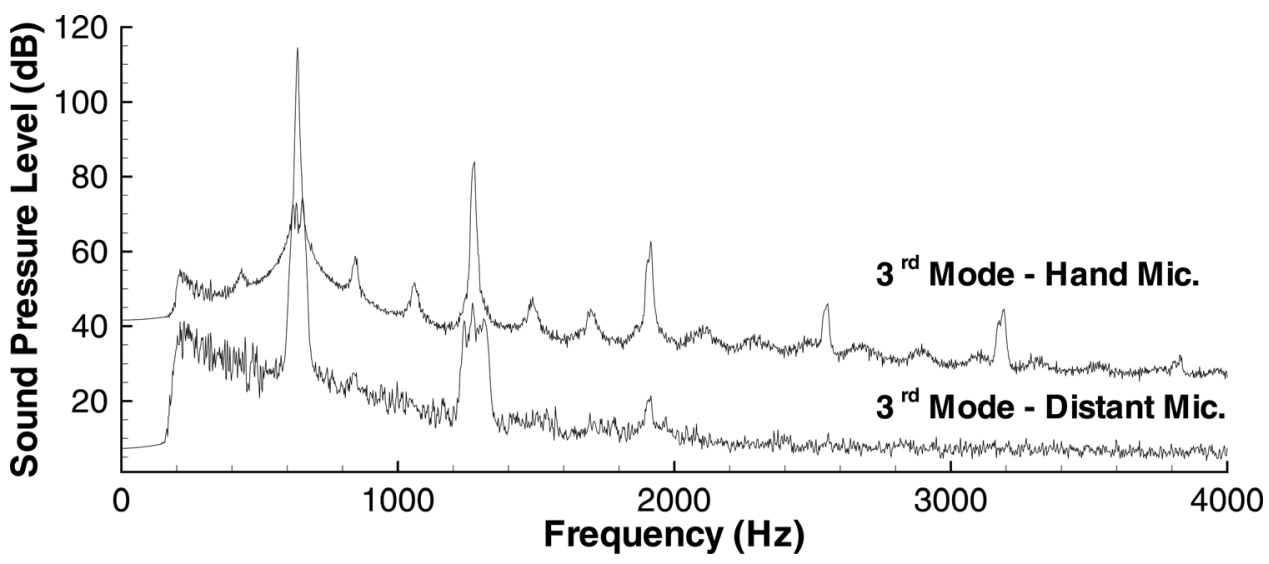

FIG. 18. Frequency spectrum plotted against the sound pressure level both for the distant microphone $(0.8 \mathrm{~m})$ and the hand microphone for the 3 rd acoustic mode.

globally resembles the measured signals. The model captures most of the modulations. It is noticeable that the estimations of the model for the listener position of $\vec{x}=(0.8 \mathrm{~m}, 0,0)$ is better than the $\vec{x}=(3 m, 0,0)$ considering both the shape of the signal and the levels of the pressure fluctuation amplitudes. This is probably due to the fact that at such a close distance from the source the radiation is dominated by the real sources $\left(S_{1}, S_{2}\right)$. At a further distance, however, not only the reflections from the floor but also other reflections from the walls that are not included in the model can be substantial.

In Fig. 22 the frequency spectra are plotted against sound pressure levels for the 3rd and 5th acoustic modes around their respective fundamental oscillation frequencies for a microphone position of $\vec{x}=(0.8 m, 0,0)$ (presented in Fig. 19) together with the estimation of the theory. For the 3rd acoustic mode, the theory agrees very well with the experiment except that there exist a stronger central peak in the theory. Since the theory assumes constant rotation speed $\Omega_{n}$, a central peak appears which is symmetric with respect to the side peaks appearing due to Doppler shift. The energy of the central peak comes from the fixed source $\left(S_{1}\right)$ and from the rotating source $\left(S_{2}\right)$ when its trajectory is not dominated by motion either toward nor away from the microphone. For the 5th acoustic mode the theory slightly underestimates the whistling frequency $(\approx 10 \mathrm{~Hz})$ and the width of the peak $(\approx 115 \mathrm{~Hz})$ compared to the experimental values.

\section{DISCUSSION}

\section{A. Missing fundamental}

A commonly observed phenomenon in short corrugated segments, e.g., Hummer, is the absence of whistling for the fundamental mode. ${ }^{3,4,18,26,28,39}$ As addressed in Sec. III C 5, the flow is probably already turbulent for the velocities where the fundamental mode is expected. Thus, it is concluded that the absence of the missing fundamental is not related to the lack of turbulence as suggested in the literature. $^{3,28}$

Experiments on the localization of the region of sound production in corrugated pipes have shown that the contribution of each cavity is not the same. ${ }^{8,12,13}$ It was demonstrated that the sound production is dominated by the cavities which are in the proximity of the acoustic pressure nodes of the standing wave along the main pipe. Considering the fundamental mode, there exist only two pressure nodes: one at the inlet and one at the outlet. Furthermore, a Hummer often has a smooth pipe segment of a few centimeters at its inlet, used to hold the pipe (Fig. 1), thus considerably decreasing the sound production capacity of the inlet section.

The developing velocity profile is another aspect that hinders the whistling for the fundamental mode. At the inlet of the Hummer a rather flat velocity profile (Fig. 10-thin) approaches to the corrugations, whereas at the outlet of the
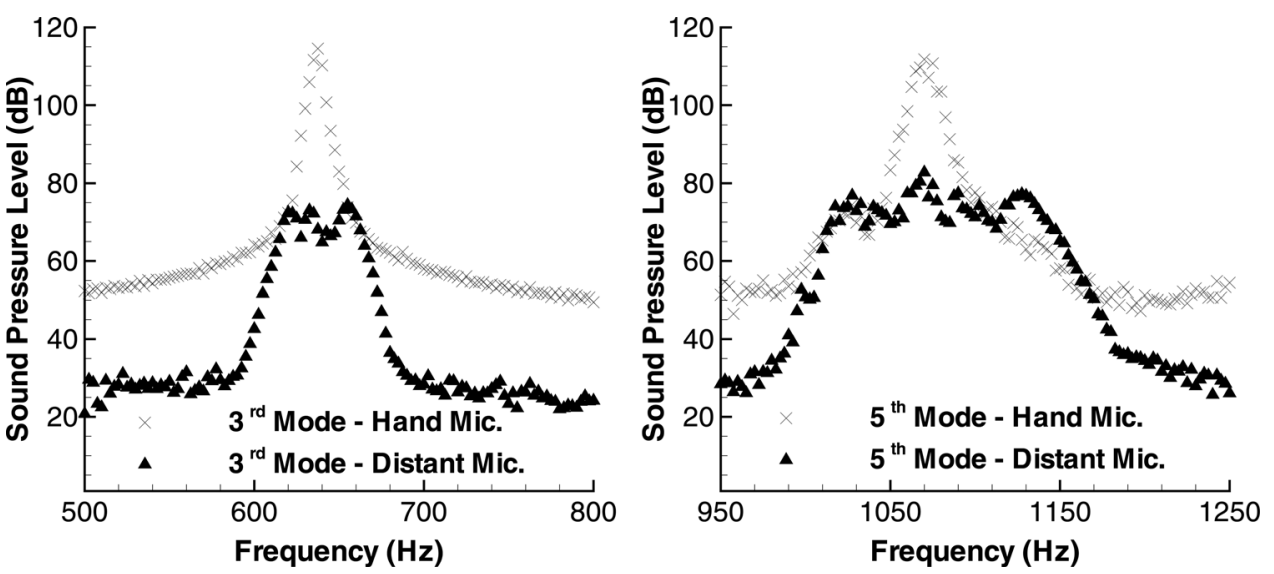

FIG. 19. Frequency spectrum plotted against sound pressure level for the 3rd and 5th acoustic modes both for the hand and the distant $(0.8 \mathrm{~m})$ microphones. 

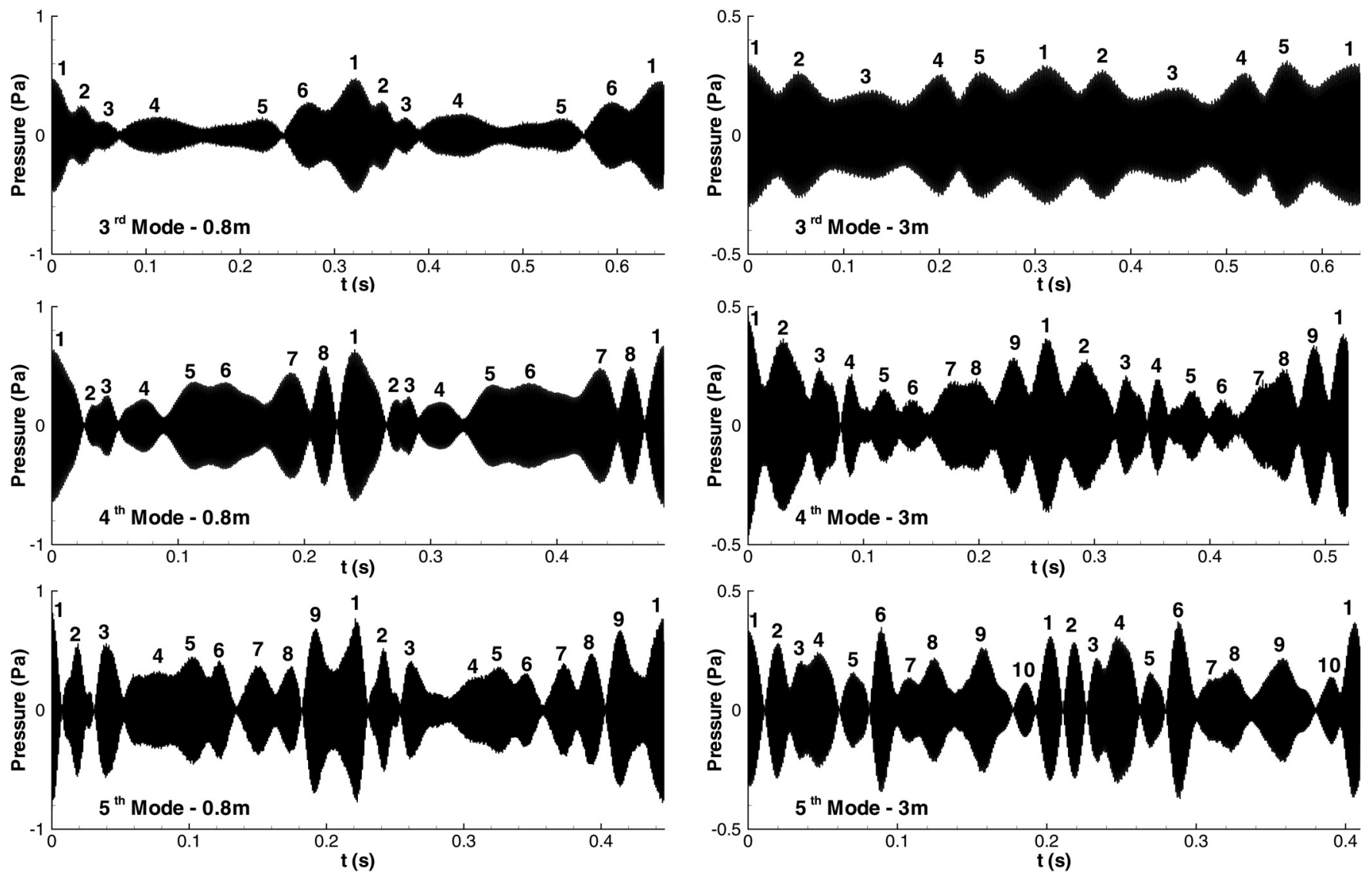

FIG. 20. Measured pressure amplitudes for listener positions of $\vec{x}=(0.8 m, 0,0)$ and $\vec{x}=(3 m, 0,0)$ as a function of time during two periods of rotation for the modes of $n=3,4$, and 5 .

pipe a fully developed velocity (Fig. 10-RANS) reaches the corrugations. As explained in Sec. IV B, different velocity profiles promotes different peak-whistling Strouhal numbers $\left(\mathrm{Sr}_{W+r_{\text {up }}}^{\mathrm{p}-\mathrm{w}}\right)$. For these two velocity profiles a difference of $50 \%$ in peak-whistling Strouhal number is predicted by the theory, as shown in Fig. 11. Thus, the source region at the inlet does not cooperate with the source region at the exit.

For these reasons the total sound source is rather weak for the fundamental mode compared to the higher modes. As a consequence the losses [Eq. (15)] become large compared to the acoustic sources and the system remains silent. This corresponds to an overshoot in Fig. 13, where the acoustic losses (horizontal line) do not intersect with an acoustic source line.

\section{B. Effect of bending}

One of the marked advantage of corrugated pipe is its ability to bend while keeping its rigidity. Thus, in various industrial applications corrugated pipes are used in a bent form. An experiment was performed with a Hummer to explore the effect of bending. The Hummer was bent at $\alpha=35^{\circ}$ in a horizontal plane, as shown in Fig. 2 . The length of the first and the second straight segments were 410 and $185 \mathrm{~mm}$, respectively. In Fig. 23 measured velocity profiles for the bent and straight Hummer are presented.

It is clear that bending has a significant effect on the velocity profile even after a straight segment of 26 corrugations
$(185 \mathrm{~mm})$. This is in agreement with the numerical simulations mentioned in Sec. III C 1, from which it is concluded that it takes 50 corrugations for the flow to reach a fully developed velocity profile.

A surprising result is that the Hummer, which was whistling (3rd acoustic mode) when it was straight, became silent in the bent configuration. A possible explanation could be found in the effect of the velocity profile on the whistling. Different velocity profiles promotes different peak-whistling Strouhal numbers as explained in Sec. IV B. Due to bending, the velocity profile approaching cavities of the Hummer is different on each side of the bend.

Consequently they have different peak-whistling Strouhal numbers and might not cooperate. Thus, they cannot produce the necessary acoustic source power for the whistling.

Although the Hummer bends during a performance due to the swinging motion, it keeps whistling. This suggest that there are more parameters involved, e.g., the angle of bending, the radius of bending, the source location with respect to the bend, etc. The importance of bending in corrugated pipe has, to the authors knowledge, not yet been addressed in the literature.

\section{Uncertainties in the radiation model}

In Sec. V A an acoustic model is proposed to estimate the radiation from a Hummer at a given listener position. 

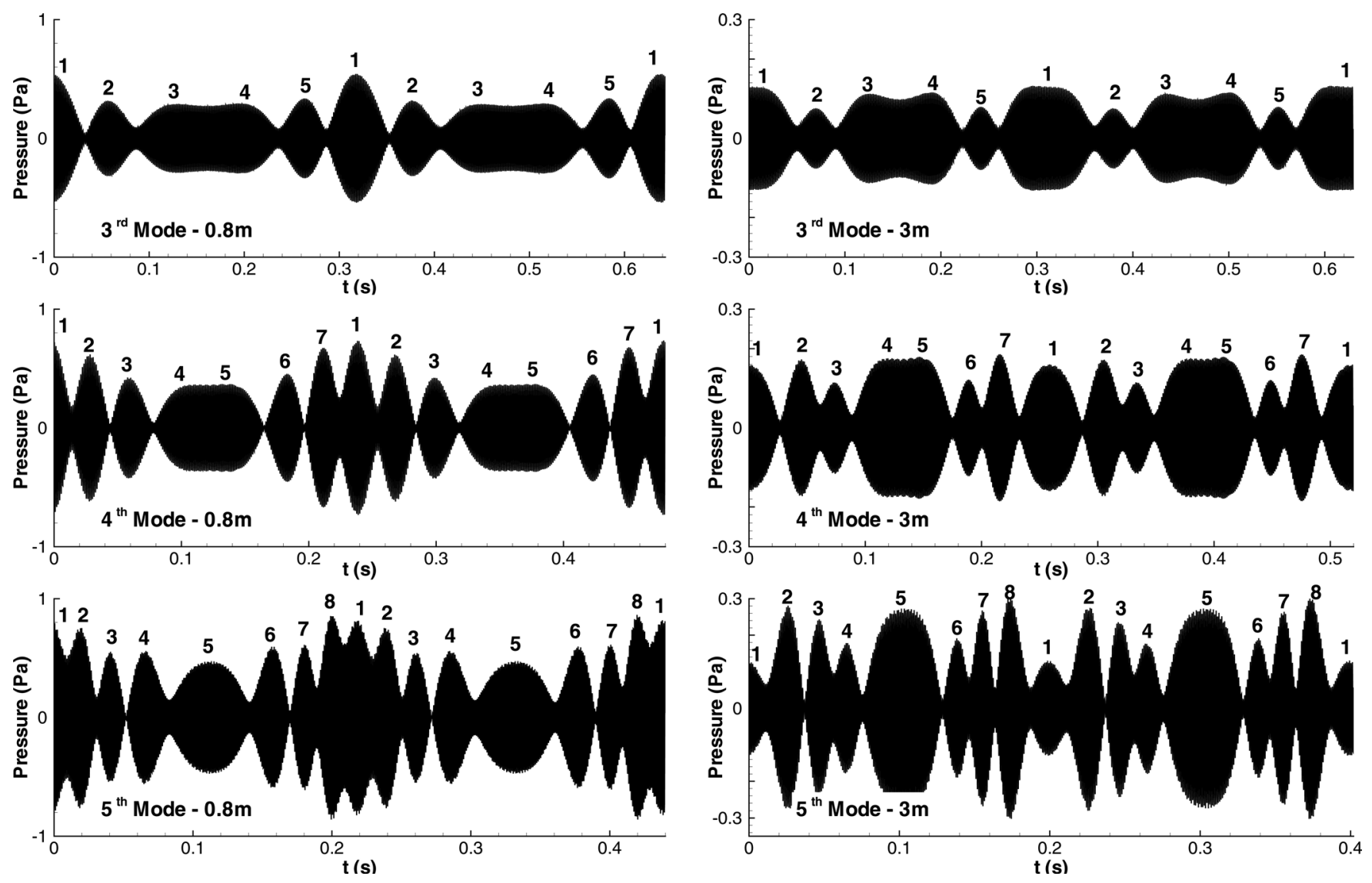

FIG. 21. Estimated pressure amplitudes from the theory [Eq. (24)] for listener positions of $\vec{x}=(0.8 m, 0,0)$ and $\vec{x}=(3 m, 0,0)$ as a function of time during two periods of rotation for the modes of $n=3,4$, and 5 .

The model, Eq. (24), uses a number of parameters with a noticeable range of uncertainty. Here these parameters are listed with respective values and the way that are estimated or assumed. It should be noted at this point that these parameters were not modified intuitively from one case to another to force a better agreement with the experimental data. The aim of the radiation study is to see how much a simple model can explain the phenomena appearing in a real performance.

The radius of rotation $(R)$, as shown in Fig. 14, is not the same as the length of the Hummer $(L)$. By using camera recordings, the radius was estimated as $80 \%$ of the pipe length for all the modes. This is a rather crude approximation. It is evident from the movies that with increasing mode number, the radius of rotation was increasing. It was, however, not included in the model.

The vertical distance between the fixed source $\left(S_{1}\right)$ and the moving source $\left(S_{2}\right)$ was taken as $h_{s}=20 \mathrm{~cm}$ for all the modes, again based on the camera recordings. Similar to the determination of radius of rotation $(R)$, this is a first order approximation and the change with the mode number is not included.

The hand holding the tube forms a flange for one of the pipe termination. This can affect the sound radiation of the fixed source, resulting in an asymmetry between the two sources. This is also not included in the radiation model and is a subject for further research.
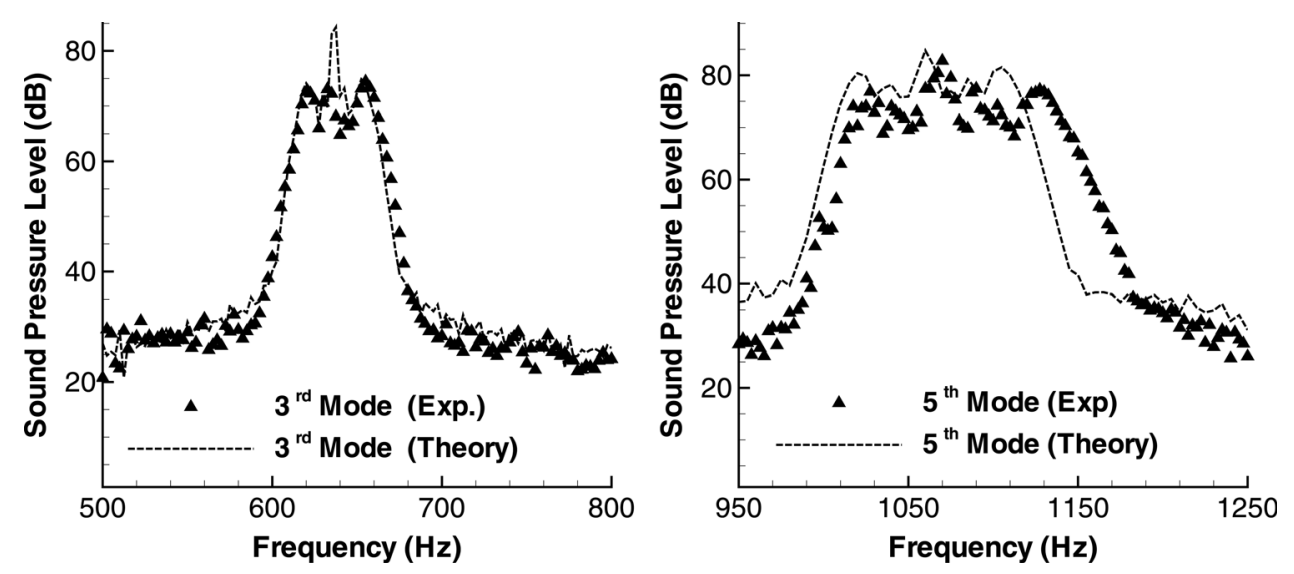

FIG. 22. Frequency spectrum plotted against sound pressure levels for the 3rd and 5th acoustic modes for a microphone position of $\vec{x}$ $=(0.8 m, 0,0)$ both measured and estimated from the theory. 


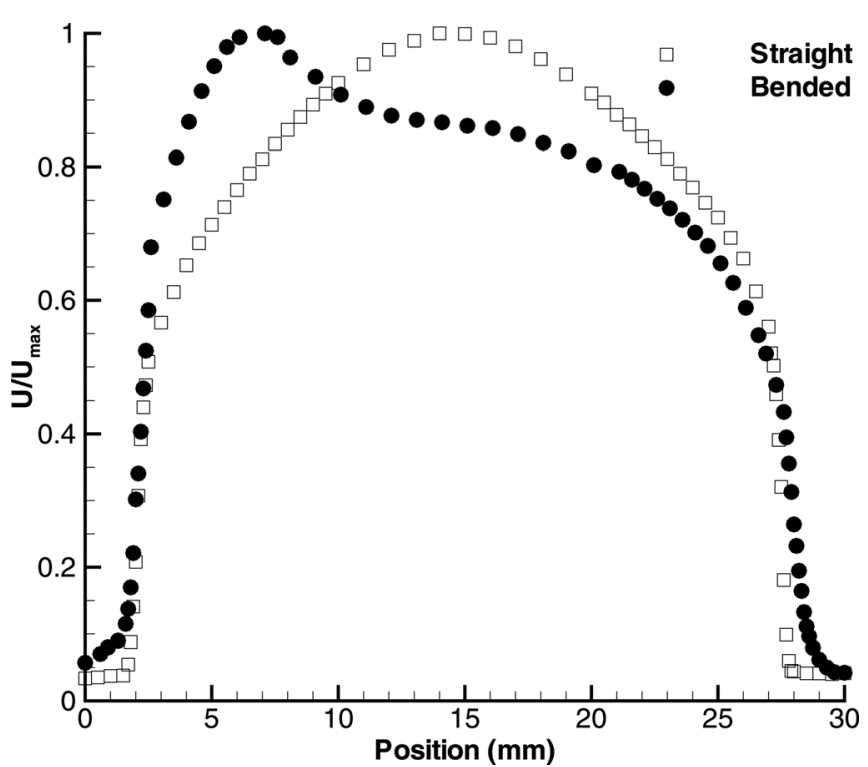

FIG. 23. Measured velocity profiles.

It was assumed in the model that the rotating source $\left(S_{2}\right)$ remains in a horizontal plane during all the performance. Yet it was apparent from the video recordings that the plane of rotation was tilted from the horizontal plane and did not preserve the same angle throughout the performance. Besides the non-constant rotation velocity $\left(\Omega_{n}\right)$, this is another cause of the asymmetry observed between the first and the second half of the period for the measured signals.

The listener positions $\vec{x}=(0.8 m, 0,0)$ and $\vec{x}=(3 m, 0,0)$ are simply the position of the audience holding the microphone at the level of the fixed source $\left(S_{1}\right)$ during the performance of the Hummer player. As a consequence the spatial position of the microphones are also prone to a significant uncertainty $( \pm 10 \mathrm{~cm})$.

\section{CONCLUSION}

In this study the sound generation in short corrugated segments used as a musical toy, e.g., Hummer, and the associate sound radiation is investigated experimentally, numerically and analytically.

Using the effective speed of sound ( $c_{\text {eff }}$ ) definition, ${ }^{18}$ the whistling frequencies $\left(f_{n}\right)$ in a corrugated pipe can be predicted within $4 \%$ (Fig. 8).

Velocity profiles measurements reveal that the fully turbulent velocity profile developed in a Hummer has a noticeably different shape than the one of a smooth pipe (Fig. 4).

Applying a numerical methodology ${ }^{7}$ based on incompressible flow simulations and vortex sound theory together with a representative velocity profile in a corrugated pipe, excellent predictions of the whistling Strouhal numbers are achieved (Fig. 12). The numerical approach combined with an energy balance can be used to estimate the acoustic fluctuation amplitudes in corrugated pipe segments, however, it should be improved before being used as a quantitative tool for the prediction of the pulsation amplitude. An accurate prediction of the whistling amplitude remains as a challenge.
Experiments indicate that the Hummer can remain silent even if the flow is turbulent. Thus, it is concluded that the absence of whistling is not related to the lack of turbulence as it has been suggested in the literature. The reason for the absence of the fundamental mode in short corrugated pipes is likely due to the lack of cooperation between the acoustic sources at the inlet and the outlet of the pipe resulting from the difference in the mean velocity profile.

An analytical radiation model is proposed in which the Hummer is modeled as two pulsating spheres: one is fixed and the other one is following a circular pattern in a horizontal plane. The model takes the reflections from the floor into account, which appears to be essential (Fig. 16). The acoustic model can predict the sound pressure level within $3 \mathrm{~dB}$ and the observed frequency at the listener position. The model can also predict qualitatively the amplitude modulation observed in the experiments (Fig. 21). It is also concluded that the amplitude modulation is mainly due to the interference between the sources.

The Doppler shift due to the rotation of the pipe outlet has a minor effect on the amplitude modulation. It has, however, a pronounced effect on the frequency, which is increasing with the increasing mode number (Fig. 20). This effect is comparable to that observed in a Leslie horn and is expected to be perceptually important.

A strong effect of bending on the whistling of a corrugated pipe has been observed, which calls for further research.

\section{ACKNOWLEDGMENTS}

The work discussed in this paper was made possible by the contributions of STW Technologiestichting (Project No. STW 08126). The authors wish to thank A. Holten, J. F. H. Willems, H. B. M. Manders, E. Cocq, and F. M. R. van Uittert for their contributions to the development of the experiments and D. Tonon, S. P. C. Belfroid, J. Golliard for the fruitful discussions. The authors appreciate the suggestion of A. J. M. Houtsma to investigate the mystery of the missing fundamental.

${ }^{1}$ S. P. Belfroid, D. P. Shatto, and R. M. Peters, "Flow induced pulsation caused by corrugated tubes," Report No. PVP2007-26503 (ASME-PVP, San Antonio, Texas, 2007).

${ }^{2}$ S. Ziada and E. T. Bühlmann, "Flow-induced vibration in long corrugated pipes, C416/010," Proceedings of the International Conference on FlowInduced Vibration, Inst. of Mech. Eng., England, 1991, pp. 417-426.

${ }^{3}$ F. S. Crawford, "Singing corrugated pipes," Am. J. Phys. 62, 278-288 (1974).

${ }^{4}$ M. Silverman and G. Cushman, "Voice of the dragon: The rotating corrugated resonator,” Eur. J. Phys. 10, 298-304 (1989).

${ }^{5}$ S. Serafin and J. Kojs, "Computer models and compositional applications of plastic corrugated tubes," Organ. Sound 10(1), 67-73 (2005).

${ }^{6}$ P. Schickele, The Definitive Biography of P.D.Q. Bach (Random House, New York, 1976), pp. 172-195.

${ }^{7}$ G. Nakiboğlu, S. Belfroid, J. Golliard, and A. Hirschberg, "On the whistling corrugated pipes: Effect of pipe length and flow profile," J. Fluid Mech. 672, 78-108 (2011).

${ }^{8}$ G. Nakiboglu, S. P. C. Belfroid, J. F. H. Willems, and A. Hirschberg, "Whistling behavior of periodic systems: Corrugated pipes and multiple side branch system,” Int. J. Mech. Sci. 52, 1458-1470 (2010).

${ }^{9}$ V. Debut, J. Antunes, and M. Moreira, "Flow-acoustic interaction in corrugated pipes: Time domain simulation of experimental phenomena," in Flow Induced Vibration, edited by I. Zolotarev and J. Horácek, 9th International Conference on Flow-Induced Vibration, Institute of Thermomechanics, Prague, Czech Republic, 2008, p. 6. 
${ }^{10} \mathrm{H}$. Goyder, "On the modelling of noise generation in corrugated pipes," J. Press. Vessel Technol. 132(4), 041304 (2010).

${ }^{11} \mathrm{M}$. Popescu and S. T. Johansen, "Modelling of aero-acoustic wave propagation in low mach number corrugated pipe flow," Prog. Comput. Fluid Dyn. 9, 417-425 (2009).

${ }^{12}$ D. Tonon, B. J. T. Landry, S. P. C. Belfroid, J. F. H. Willems, G. C. J. Hofmans, and A. Hirschberg, "Whistling of a pipe system with multiple side branches: comparison with corrugated pipes," J. Sound Vib. 329, 1007-1024 (2010).

${ }^{13}$ J. Golliard, D. Tonon, and S. P. C. Belfroid, "Experimental investigation of the source locations for whistling short corrugated pipes, FEDSMICNMM2010-30732," Proceedings of ASME 2010 3rd Joint US-European Fluids Engineering Summer Meeting and 8th International Conference on Nanochannels, Microchannels and Minichannels, Montreal, Canada, 2010.

${ }^{14}$ H. Schlichting, Boundary Layer Theory, 7th ed. (McGraw Hill, New York, 1979), pp. 127-130.

${ }^{15}$ N. Curle, "The influence of solid boundaries upon aerodynamic sound," Proc. R. Soc. London, Ser. A 231, 505-514 (1955).

${ }^{16}$ N. H. Fletcher and T. D. Rossing, The Physics of Musical Instruments (Springer-Verlag, New York, 1991), pp. 436-447.

${ }^{17}$ A. H. Shapiro, The Dynamics and Thermodynamics of Compressible Fluid Flow (Ronald, New York, 1953), pp. 92-93.

${ }^{18} \mathrm{~J}$. W. Elliott, "Corrugated pipe flow," in Lecture Notes on the Mathematics of Acoustics, edited by M. C. M. Wrigth (Imperial College Press, London, 2005), pp. 207-222.

${ }^{19}$ S. V. Patankar and D. B. Spalding, "A calculation procedure for heat mass and momentum transfer in three dimensional parabolic flows," Int J. Heat Mass Transfer 15, 1787-1806 (1972).

${ }^{20}$ R. D. Blevins, Applied Fluid Dynamics Handbook (Krieger, Malabar, FL, 1984), pp. 55-56.

${ }^{21}$ C. J. Nederveen, Acoustical Aspects of Woodwind Instruments (Northern Illinois University Press, DeKalb, IL, 1998).

${ }^{22}$ J. C. Bruggeman, "The propagation of low-frequency sound in a twodimensional duct system with t-joints and right angle bends: Theory and experiment," J. Acoust. Soc. Am. 82, 1045-1051 (1987).

${ }^{23} \mathrm{~V}$. Sarohia, "Experimental investigation of oscillations in flow over shallow cavities," AIAA J. 15(7), 984-991 (1977).

${ }^{24}$ S. Ziada, H. Ng, and C. E. Blake, "Flow excited resonance of a confined shallow cavity in low mach number flow and its control," J. Fluids Struct. 18, 79-92 (2003).
${ }^{25}$ J. C. Bruggeman, A. Hirschberg, M. E. H. vanDongen, A. P. J. Wijnands, and J. Gorter, "Self-sustained aero-acoustic pulsations in gas transport systems: experimental study of the influence of closed side branches," J. Sound Vib. 150, 371-393 (1991).

${ }^{26}$ Y. Nakamura and N. Fukamachi, "Sound generation in corrugated tubes," Fluid Dyn. Res. 7, 255-261 (1991).

${ }^{27}$ U. R. Kristiansen and G. A. Wiik, "Experiments on sound generation in corrugated pipes with flow," J. Acoust. Soc. Am. 121, 1337-1344 (2007).

${ }^{28}$ L. H. Cadwell, "Singing corrugated pipes revisited," Am. J. Phys. 62, 224-227 (1994).

${ }^{29}$ M. T. Angus and M. T. Lyon, Quiet gas connector, United States Patent Application Publication No. US20080012331A1, 2008.

${ }^{30} \mathrm{~S}$. Ziada and S. Shine, "Strouhal numbers of flow-excited acoustic resonance of closed side branches," J. Fluids Struct. 13, 127-142 (1999).

${ }^{31}$ P. Martínez-Lera, C. Schram, S. Föller, R. Kaess, and W. Polifke, "Identification of the aeroacoustic response of a low mach number flow through a t-joint," J. Acoust. Soc. Am. 126(2), 582-586 (2009).

${ }^{32}$ A. M. Binnie, "Self-induced waves in a conduit with corrugated walls. II. Experiments with air in corrugated and finned tubes," Proc. R. Soc. London, Ser. A 262, 179-191 (1961).

${ }^{33} \mathrm{U}$. Ingard and V. K. Singhal, "Effect of flow on the acoustic resonances of an open-ended duct," J. Acoust. Soc. Am. 58(4), 788-793 (1975).

${ }^{34}$ A. D. Pierce, Acoustics: An Introduction to Its Physical Principles and Applications (Acoustical Society of America, New York, 1989), pp. 210, 211.

${ }^{35}$ A. P. Dowling and J. F. Williams, Sound and Sources of Sound (Ellis Horwood, Ltd., West Sussex, England, 1983), pp. 187-190.

${ }^{36} \mathrm{~S}$. W. Rienstra and A. Hirschberg, Lecture Notes: An Introduction to Acoustics (Eindhoven University of Technology, Eindhoven, Netherlands, 2004), pp. 278-292.

${ }^{37}$ J. Smith, S. Serafin, J. Abel, and D. Berners, "Doppler simulation and the Leslie," Proceedings of the 5th International Conference on Digital Audio Effects (DAFx-02), Hamburg, Germany, 2002.

${ }^{38}$ R. Kronland-Martinet and T. Voinier, "Real-time perceptual simulation of moving sources: Application to the leslie cabinet and $3 \mathrm{~d}$ sound immersion," EURASIP J. Audio Speech Music Process. 2008, 849696 (2008).

${ }^{39} \mathrm{P}$. Cermak, "Über die Tonbildung bei Metallschläuchen mit Eingedräcktem Spiralgang (On the sound generation in flexible metal hoses with spiraling grooves)," Phys. Z. 23, 394-397 (1922). 